\title{
Recent developments in the chiroptical properties of chiral plasmonic gold nanostructures: bioanalytical applications
}

\author{
Nebu John ${ }^{1} \cdot$. Anslin Thankachan Mariamma ${ }^{2}$
}

Received: 14 June 2021 / Accepted: 14 October 2021 / Published online: 22 November 2021

(c) The Author(s), under exclusive licence to Springer-Verlag GmbH Austria, part of Springer Nature 2021

\begin{abstract}
The presence of excess L-amino acid in the Murchison meteorite, circular polarization effect in the genesis of stars and existence of chirality in interstellar molecules contribute to the origin of life on earth. Chiral-sensitive techniques have been employed to untangle the secret of the symmetries of the universe, designing of effective secure drugs and investigation of chiral biomolecules. The relationship between light and chiral molecules was employed to probe and explore such molecules using spectroscopy techniques. The mutual interaction between electromagnetic spectrum and chirality of matter give rise to distinct optical response, which advances vital information contents in chiroptical spectroscopy. Chiral plasmonic gold nanoparticle exhibits distinctive circular dichroism peaks in broad wavelength range thereby crossing the limits of its characterization. The emergence of strong optical activity of gold nanosystem is related to its high polarizability, resulting in plasmonic and excitonic effects on incident photons. Inspired by the development of advanced chiral plasmonic nanomaterials and exploring its properties, this review gives an overview of various chiral gold nanostructures and the mechanism behind its chiroptical properties. Finally, we highlight the application of different chiral gold nanomaterials in the field of catalysis and medical applications with special emphasis to biosensing and biodetection.
\end{abstract}

Keywords Gold nanostructures · Chirality · Circular dichroism · Plasmonic nanoparticle · Surface plasmon resonance · Chiroptical properties

\section{Introduction}

Chirality is a fascinating property of certain objects in nature [1]. Chirality means handedness, is observed in snail shells, gourd tendrils, human hands and even spiral galaxies [2,3]. An object is said to be chiral if it is not superimposable on its mirror image [4]. Chirality plays a major role in the origin of life. The building blocks of life such as amino acids, carbohydrates and nucleic acids are chiral [5, 6]. Deoxyribonucleic acid/ribonucleic acid (DNA/RNA) consists of

Nebu John

nebujohnj@gmail.com

Anslin Thankachan Mariamma

anslintm@gmail.com

1 The Post Graduate and Research Department of Chemistry, Mar Thoma College, Mahatma Gandhi University, Tiruvalla 689103, Kerala, India

2 The Post Graduate Department of Mathematics, St. Gregorios College, University of Kerala, Kottarakara 691531, Kerala, India residues with the same handedness owing to act as template in replication of cell division process. In prebiotic chemical world, these macromolecules synthesized from small molecules are prone to have existed as racemic mixtures [5]. Life on earth manifests a priority for L-amino acids and D-sugars, in spite of a thermodynamic stability equilibrium of the $\mathrm{L}$ - and D-enantiomers of these molecules. The bioactivities of a chiral molecule depend on its handedness; for example, one enantiomer might act as a drug while the other might be toxic [7]. Accordingly, chirality of molecules has a vital role in functionality and interaction with other molecules.

Chirality is an intrinsic property of certain molecules or nanostructures, which on interaction with light, generate a significant contribution to both absorption and scattering $[1,8]$. The interaction of chiral molecules with circularly polarized photons, circular dichroism (CD) effect occurs relating the variation in molecular absorption of clockwise and counter-clockwise circularly polarized lights [9]. CD spectroscopy is a potential technique to unravel molecular chirality and conformational analysis in a chemical reaction [10]. The chiroptical response of a chiral ensemble 
can be tuned and enhanced by the integration of plasmonic nanoparticles [11]. Chirality existence in nanosystem taking place via (i) intrinsic chirality depends on the geometry of the particle (ii) incorporation of chiral templates like DNA and peptides and (iii) plasmon-induced chirality [12]. Nanoparticles with chiral plasmonic properties generate chiroptical activity owing to its ability to create broadband circular polarization states of photons [13]. The origin of chirality in nanoparticle is well investigated using plasmonic chiral gold nanoparticles (Au NPs). The interaction of electronic states of ligands with the surface gold atoms give rise to distinct chirality effects in chiral Au NPs [14].

In this review, we present the origin of chirality of gold nanostructures, different types of chiral gold nanostructures, enhancement of chirality in Au NPs and its applications in physical, chemical and biological fields. The distinct electromagnetic properties, shift in wavelengths, energy changes associated with Au NPs and hybrid plasmonic Au NPs are also illustrated. We concentrate our attention on the phenomena of electronic interactions between ligands and surface gold atoms, variation in optical properties such as plasmonic resonance and $\mathrm{CD}$. The mechanism of innate chirality and external factors contributing for chirality of gold nanostructures were also studied. Finally, we provide an outlook for numerous bioanalytical applications based on plasmonic chiral gold nanostructures.

\section{Chiral gold nanostructures}

A chiral dimer of gold nanorods (Au NRs) could be synthesized by using a chiral-binding agent between the adjacent nanorods in a solution. The size parameter and aspect ratio of the Au NRs affect its optical activity. Exciton coupling theory highlights the dipole-dipole interaction between dimer of Au NRs arranged as prolate ellipsoids in a medium of refractive index $n=1.5$. The energy level of the localized surface plasmon resonance (LSPR) consists of hybrid modes such as symmetric $\left(\omega_{+}\right)$and antisymmetric $\left(\omega_{-}\right)$. The scaling of the system increased the extinction efficiency by a factor of 6 , and overall extinction efficiency of the longitudinal LSPR contributes to the same extent of absorption and scattering. The asymmetric $\mathrm{CD}$ spectrum appears due to the retardation effect in dimers, where the electromagnetic coupling depends on the wavelength of the incident light. The CD efficiency of the chiral Au NR dimer varies in a nonlinear basis with the aspect ratio of the Au NR. The enhancement of CD signal depends on interparticle distance, size of the particle, cooperative activity between neighbouring multiple Au NRs and higher-order multipolar effect. Like molecular system, plasmonic structure gives rise to a dark mode in addition to the bonding mode if the dipoles remain parallel to each other. The integrated intensity of the antibonding mode (dark mode) is the same as that of the bonding mode [15].

The geometrical chirality in left- and right-handed gold Gammadions produces strong plasmonic CD bands. The $\mathrm{CD}$ response of the Gammadions establishes that its origin is either due to sum of the localized response from different regions in the structure or due to collective response of the overall structure. The left- and right-handed chiral gold Gammadions show opposite signal in the spatial dissymmetry maps. The Gaussian-like distribution based on the geometric centre of Gammadions is in good agreement with simulated spatial dissymmetry maps. The dissymmetry sign switches between left- and right-handed 150-nm Gammadion in transmission mode at $600 \mathrm{~nm}$. The Gaussian response of the Gammadions establishes that its origin is either due to sum of the localized response from different regions in the structure or due to collective response of the overall structure [16].

Gold nanodumbbells (Au NDs) were dimerized as parallel, twisted and twisted with planar displacement (Fig. 1a). Transmission electron microscopy (TEM) images of the $\mathrm{Au}$ NDs depict that the particles have smaller aspect ratio (Fig. 1b) and remain in different configurations (Fig. 1c). The UV-visible extinction spectroscopy of the Au NDs gives a red shift after the formation of dimers (Fig. 1d and e). The red shift in the transverse mode as well as in the longitudinal mode can be attributed to the side-by-side assembly of the dimer, large refractive index of the adsorbed polystyrene and the interaction of plasmon resonances to changes in the refractive index. The absence of detectable $\mathrm{CD}$ signal before and after dimerization of Au NDs, owing to the existence of racemic mixture of chiral dimers in solution (Fig. 1f and g) [17].

The DNA-driven nanoparticle chiral pyramids comprising of Au NPs, CdSe@ZnS quantum dots and silver nanoparticles (Ag NPs) give CD signals in a wide range from 350- to 550-nm region. Chiral Au NPs synthesized using D-glutamic acid and L-glutamic acid inhibit the formation of human serum albumin fibrils. The surface chirality of Au NPs strongly influences the fibrillation process, which is more for D-glutamic acid-based Au NPs [18]. The $\mathrm{Au}_{144}(\mathrm{SR})_{60}$ with chiral icosahedral shell $\left(1-\mathrm{Au}_{60}\right)$ have unique optical, electronic, vibrational and structural properties [19].

The chiral handedness of Au NRs obtained from the helical glutathione (GSH) oligomers result in the formation of end-to-end crossed (EEX) Au NR junctions [20]. Peptide conjugates and alkyne-terminated aliphatic substrates $\mathrm{C}_{18^{-}}\left(\mathrm{PEP}_{\mathrm{Au}}^{\mathrm{M}-\mathrm{ox}}\right)_{2}$ direct the formation of plasmonic chiroptically active single helical Au NPs, where peptide conjugate $\mathrm{PEP}_{\mathrm{Au}}{ }^{\mathrm{M}-\mathrm{ox}}=\mathrm{AYSSGAPPM}^{\mathrm{ox}} \mathrm{PPF}$, $\mathrm{C}_{18}=18$ carbon chain and ox $=$ oxidation [21]. The chirality of Au NRs depends on the aspect ratio. The chirality 
Fig. 1 Structural and spectral characteristics of Au NDs and Au ND dimers. a Cartoon depiction of the dimer formation scheme through polymer capture, resulting in parallel (top), twisted (middle) and twisted with planar displacement (bottom) dimers. b, c TEM images of the Au NDs before and after dimerization, respectively. Scale bars are $100 \mathrm{~nm}$. d, e Ensemble UV-Vis extinction spectra of the Au ND and dimer samples, respectively. The Au NDs show maxima at 521 and $812 \mathrm{~nm}$. The maxima for Au ND dimers are at 534 and $816 \mathrm{~nm}$. f, $\mathbf{g}$ Ensemble CD spectra of the Au ND and dimer samples, respectively. No peaks are observed in either case. "Reprinted with permission from Ref. 17. Copyright (2016) American Chemical Society"

a)

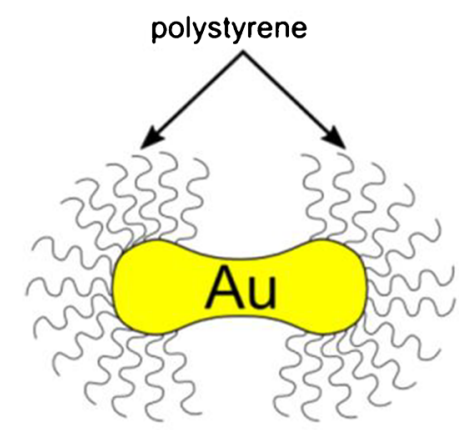

THF/DMF b)

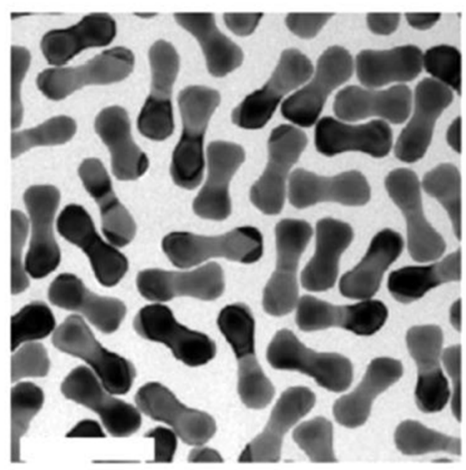

d)

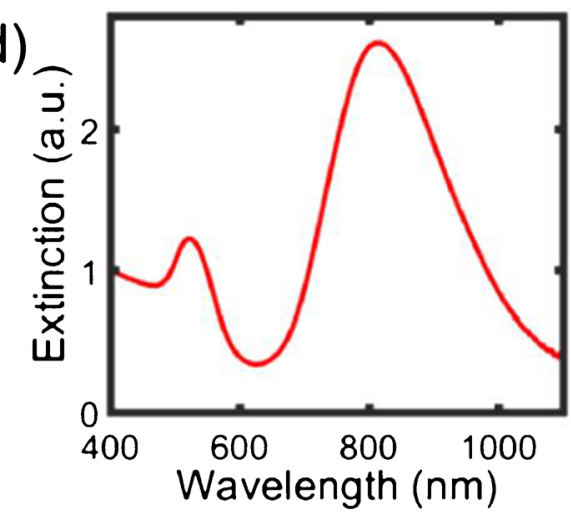

f)

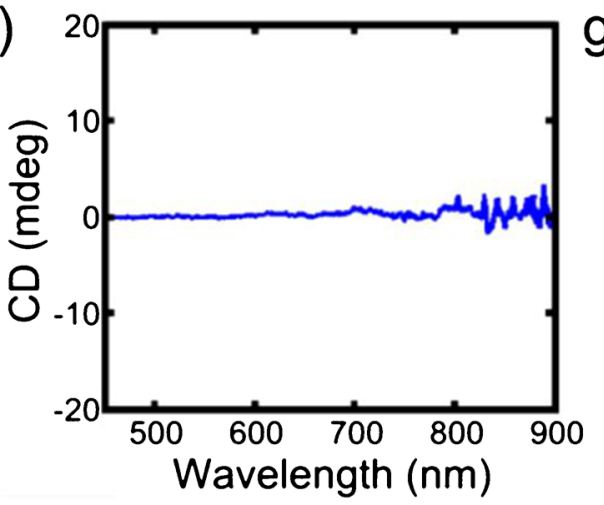

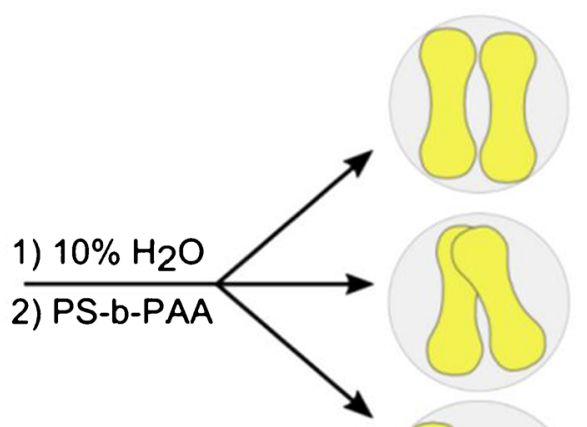

Q

c)

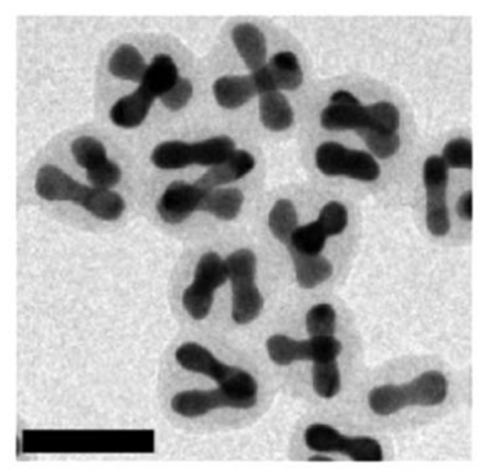

e)

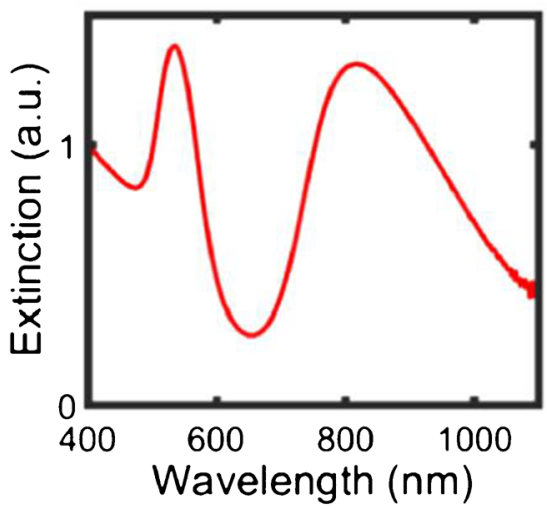

g)

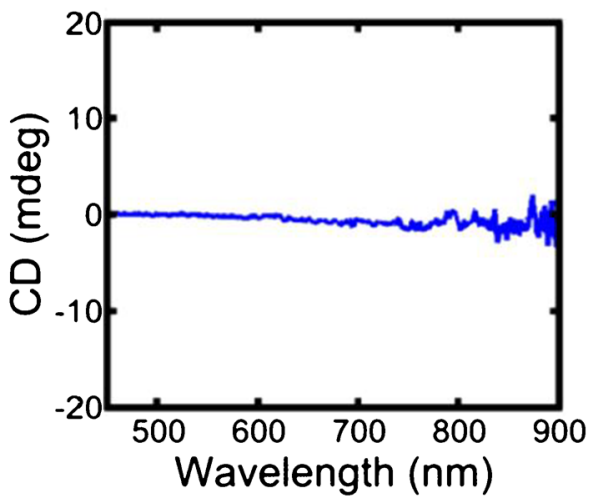




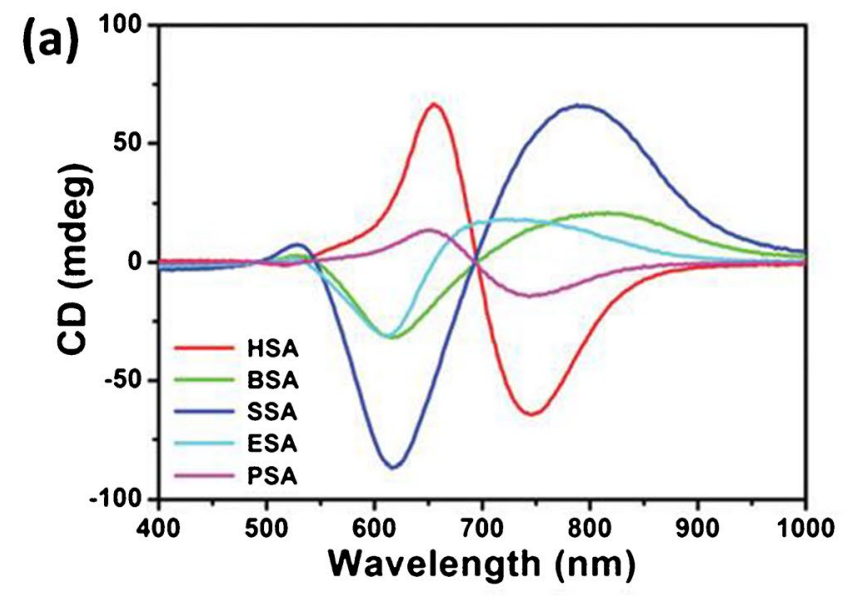

(c)

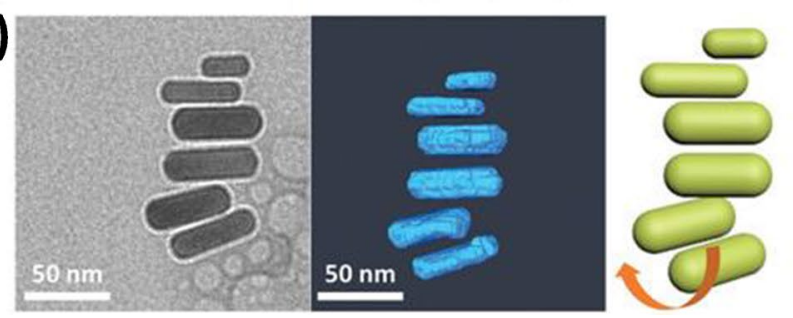

Fig. 2 Vis-NIR region. $\mathbf{a} C D$ and $\mathbf{b}$ UV-Vis absorbance spectra of five SA-guided GNR assemblies; the cryo-TEM (left), 3D reconstruction (middle) and schematic images (right) of the nanoassemblies guided by HSA (c) and BSA (d). The GNR, CTAB and SA concen-

increases with increase in aspect ratio. It depends upon the concentration of $\mathrm{AgNO}_{3}$ in growth solution. The length of Au NRs increases, whereas diameter decreases with increase in concentration of $\mathrm{AgNO}_{3}$. The origin of intrinsic chirality in nanocrystals is due to the point defects, dislocation and chiral defects in the nanosystem, which is greater in Au NRs with high aspect ratio. Here Au NR-based colorimetric assay was used for the detection of L-valine in the presence of $\mathrm{D}$-valine with a detection limit of $0.1 \mathrm{mM}$ [22]. The human serum albumin and porcine serum albumin-guided chiral assembly of Au NRs exhibit left-handed CD responses, whereas equine serum albumin, sheep serum albumin and bovine serum albumin executed on the converse (Fig. 2a). The interaction of albumin with Au NRs is electrostatic. The cryogenic TEM image of human serum albumin-guided and bovine serum albumin-guided Au NRs manifested left-handed and right-handed twisted side-by-side geometry respectively (Fig. 2c and d). These spatial asymmetry geometries indicated the template-dependent chirality of serum albumins. The handedness of the chiroptical properties depends on the surface charge distributions of the serum albumins and the ambient $\mathrm{p}^{\mathrm{H}}[23]$. (b)

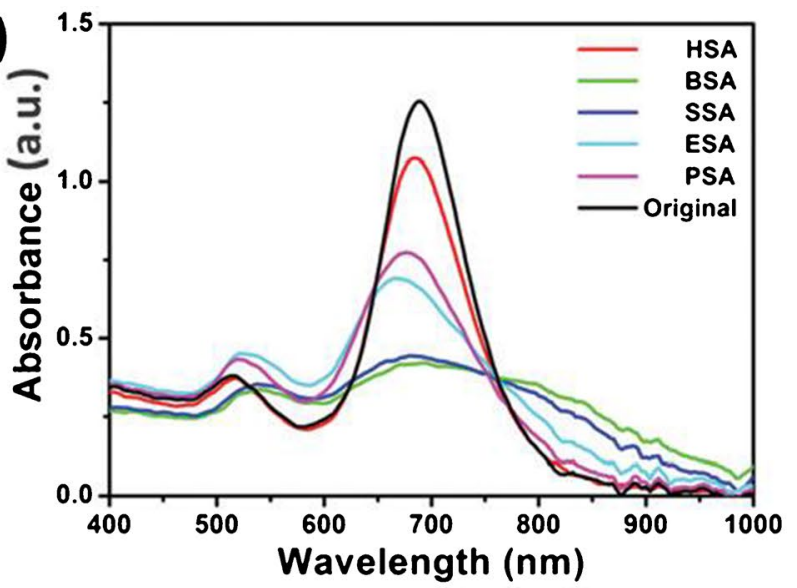

(d)

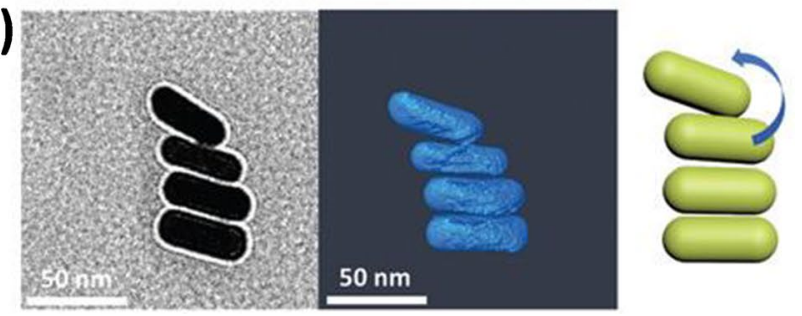

trations, $\mathrm{pH}$ value and ionic strength of each individual assembly system are all set to $5.0 \mathrm{nM}, 2.0 \mathrm{mM}$ and $3.0 \mathrm{mM} ; 7.4$; and $10.0 \mathrm{mM}$, respectively. Reproduced from Ref. 23 with permission from the Royal Society of Chemistry"

\section{Chiral plasmonic gold nanoparticle}

A multi-chirality evolution step method for the synthesis of chiral plasmonic Au NPs with increase in particle uniformity has been utilized for the enhancement of chiroptic response. The Au NPs having 432 point-group symmetry (432 helicoid 111) shows well-defined gaps between the nanoparticle, increased dis-symmetry factor of $g=0.31$ and distinct cubic boundaries [24]. Chiral Au NPs are produced by using peptides with random coil and $\alpha$-helix. These Au NPs show CD signals at the surface plasmon resonance (SPR) wavelength $t$ $520 \mathrm{~nm}$ ) and in the visible region of spectrum get red-shifted on aggregation [25]. A core-satellite nanostructure of $\mathrm{Au}$ NRs (core) and Au NPs (satellites) were created using DNA as linkers. CD spectrum displayed plasmonic CD (PCD) corresponding to $\mathrm{Au}$ NRs and Au NPs at SPR wavelength region (Fig. 3). The chirality depends upon the number of $\mathrm{Au}$ NPs around Au NRs, structure of core-satellites nanosystem, ligand induction, dipole-dipole interactions and chiral arrangement of surface atoms or molecules [26].

Chiral cholesterol-capped Au NPs were constructed and synthesized uniformly dispersed mixtures in different nematic liquid crystal hosts. These nanoassemblies prepared in the presence of disulphide chiral bias are strong chiral inducers as compared to that in the absence of a chiral bias [27]. The chiral discrimination of tyrosine and phenylalanine 
Fig. 3 Representative TEM images of the assemblies for different hybridization times; the assembled times were $\mathbf{a} 5$ and b 30 min and $\mathbf{c} 1, \mathbf{d} 3$, e 6 and $\mathbf{f}$ $12 \mathrm{~h}$. "Reprinted with permission from Ref. 26. Copyright (2013) American Chemical Society"

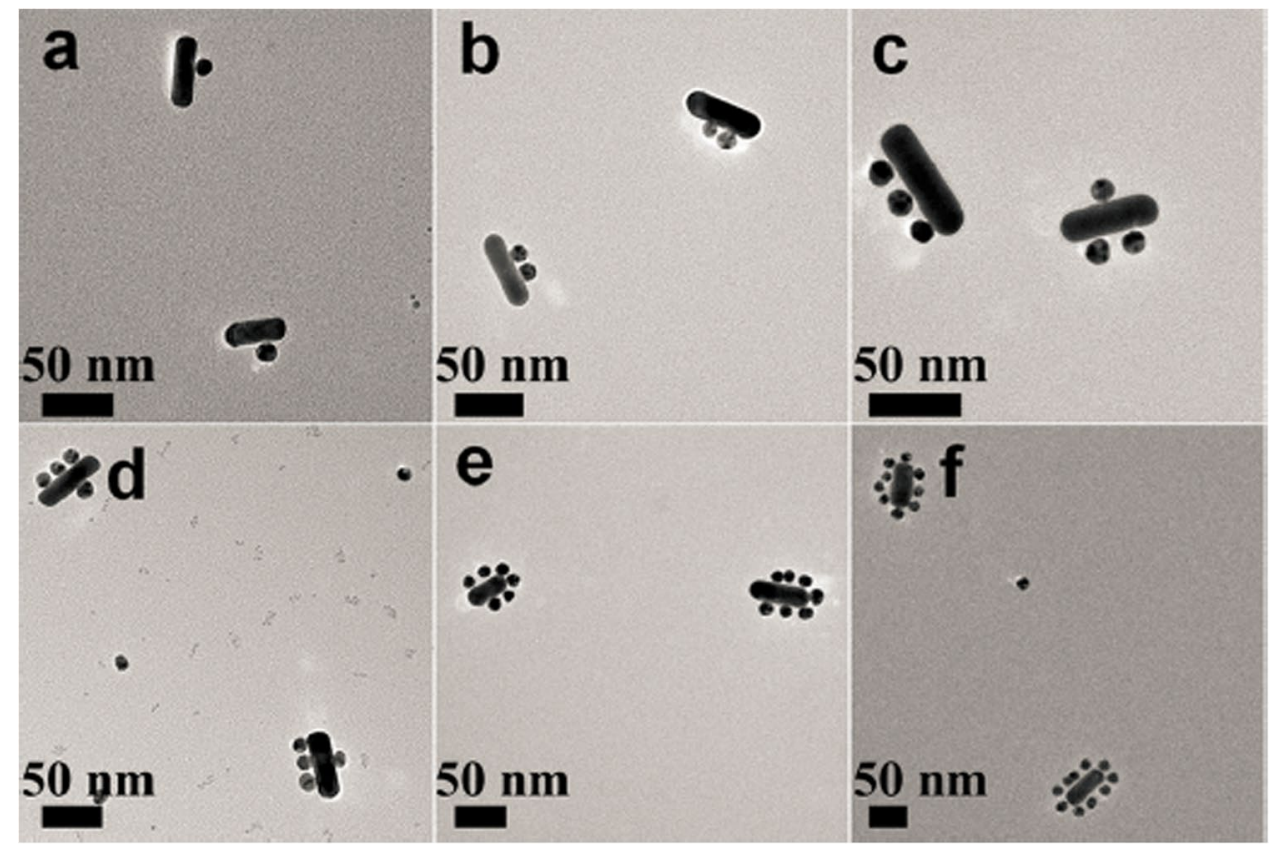

enantiomers with cysteine-modified Au NPs was analysed with Raman spectra. It was found that the left-handed enantiomer combination of tyrosine or phenylalanine with same handedness cysteine-based Au NPs gives enhanced Raman signals. Similar observation was obtained for D-enantiomer as well. This type of interaction occurs due to (i) the presence of three interaction sites in the vicinity of D-selector and D-enantiomer of L-selector and L-enantiomer and (ii) possibility of more hydrogen bonds between different amino acids of same handedness as that of different handedness [28]. The blending of the results obtained from CD spectra and Raman spectra can be used for the discrimination of enantiomers of amino acids. Chiral liquid crystalline Au NPs (LC-Au NPs) were assembled into a fluid lamellar structure showing chiroptical activity. The arylamine in liquid crystal ligands reduce $\mathrm{Au}(\mathrm{III})$ to $\mathrm{Au}(0)$ and bind to the Au NPs core. The LC-Au NPs exhibit temperature-dependent bisignate CD signals. The thick coating of Au NPs by mesogens produces a negative refractive index. This physical quantity can be utilized for the investigation of metamaterials [29].

\section{Chiral plasmonic gold nanoantenna}

The combination of Au NRs with a 3D chiral structure known as nanoantenna delivers powerful chirality probes for functionalization with proteins, DNA and other metal nanoparticles. The ordering of gold nanorods on a fibre backbone gives a three-dimensional chiral structure known as plasmonic gold nanoantenna. The fluid dispersion of chiral fibres is obtained by adding water in anthraquinonebased oxalamide-1 present in DMF/ethanol solution. This plasmonic gold nanoantenna generates strong surface plasmon-mediated circular dichroism (SP-CD) with high anisotropy factors (g-factor) across visible and near-infrared wavelengths (600-900 nm). These Au NRs in fluid suspension manifest a g-factor of 0.022 , which is comparable to proteins complexes (0.06) and polyaromatic compounds (0.05). The SP-CD band arises as a result of 3D chiral arrangement of the Au NRs, and the intensity of SP-CD decreases with an increase in disorder of the particle. Here, each particle is considered as an electric dipole where the SP-CD depends on the geometry and composition of the structure [30].

Plasmonic helical nanoantenna on thin gold layer converts subwavelength longitudinal near-fields into circularly polarized wave and vice versa. The nanoantenna consists of L-handed four-turn Au-coated carbon helix coated on cylindrical pedestal. The excitation of an L-handed helix with circular polarization generates a longitudinally oriented dipole containing the surface plasmons. This effect occurs in a broad wavelength range owing to the waveguide mode of surface plasmons propagating along the nanoantenna [31]. The stability of such nanoantenna is excellent. The nature of nanoantenna with right-handedness upon excitation could also contribute to its usage in practical applications.

\section{Hybrid chiral gold nanostructures}

The chiral gold-upconversion nanoparticle pyramids exhibit strong plasmonic circular dichroism at $512 \mathrm{~nm}$ and luminescence in the range 500-600 $\mathrm{nm}$. The resulting nanopyramids enumerate the following process: (i) bathochromic shift in UV-Visible spectrum, (ii) strong CD signal and (iii) luminescence resonance energy transfer (LRET) from 
upconversion nanoparticle to Au NPs. The merit of this nanoassembly is that multiple optical on-off changes take place on interaction with active analytes [32]. The chiral gold nanoparticle-chromophore hybrid complexes possess tuneable strong chiroptical activity. The chiroptical activity is tuned by optimizing the spectral overlap between plasmon resonances and chiral collective molecular excitations. The chiral Au NP-chromophore hybrid assembly with the aid of DNA-template establishes a spectral overlap between plasmons and collectively coupled chiral excitons. This leads to an enhancement in molecular $\mathrm{CD}$ along with a triggered plasmonic $\mathrm{CD}$. This nanosystem can act as a bisignate $\mathrm{CD}$ probe for nanosensing strategies [33].

\section{Core-satellite/shell chiral gold nanostructures}

The chiral DNA-modified shell core-satellite nano superstructures based on Au NPs have been self-assembled for the detection of mycotoxin. The shell core structure consists of $\mathrm{Au}-\mathrm{Ag}$ alloyed hollow sphere as core with a thin layer of gold as outer shell. The core-satellite structure was developed with the dipole-dipole interaction between DNAmodified shell core structure and Au NPs, which produce a strong chiral signal [34]. The gold/silver core shell nanorods functionalized with DNA has enhanced plasmonic properties and exhibit increased circular dichroism responses. The Ag shell thickness can be used as a parameter for tuning the LSPR. The DNA origami platform holds great promise for synthesizing bimetallic nanoparticles [35]. The chiral core-satellite silver nanorod (Ag NR)-gold nanoparticle nanostructure has been fabricated by using L-cysteine and D-cysteine as bridging molecules. The L- and D-cysteinebased core-satellite structure gives mirrored CD signals. Increase in concentration of both enantiomers produces a red shift in CD spectra. Here, the origin of optical activity is explained as (i) electronic interaction between chiral cysteine molecules and achiral metal electrons and (ii) vicinal effects developed from the dissymmetric fields via the formation of chemical bond between dissymmetric centre and the metal core.[36].

The chiral cysteine molecules intercalated between $\mathrm{Au}$ NR core and Au shell, which is modified with cetyltrimethylammonium bromide and cetylpyridinium chloride (Fig. 4a). The overall morphology of the core-shell structure consists of an additional arrow head-like end cap as that of Au NR core (Fig. 4b). The modification of the core-shell structure with CTAB imparts positive charge on its surface. The trisodium citrate molecules link the modified core-shell assembly side-by-side (Fig. 4c and d). The surfactants transfer and amplify the chirality induced by the adsorbed chiral molecule. The chiral force developed, owing to the formation of hot spots via dipole-dipole interaction between chiral molecule and achiral plasmonic gold nanostructures [37].
The surfactants and chiral thiols could induce strong chiroptical effects in gold-nanoparticle based core-shell structures.

\section{DNA-based chiral gold nanostructures}

Three-dimensional (3D) plasmonic chiral gold nanostructures were developed through programmable transformation of Au NPs using DNA origami as template. A twodimensional (2D) DNA origami template organized the Au NPs into a 3D helix with engineerable optical chirality [38]. The plasmonic coupling between Au NRs in a pattern is arranged in the presence and absence of a gold nanosphere using DNA origami. Here, the gold nanosphere acts as a transmitter. The two Au NRs having 54-nm length and 23-nm width are separated by a surface-to-surface distance of $62 \mathrm{~nm}$ and tilted by $90^{\circ}$ to each other. These nanosystems were assembled in thiol-functionalized DNA (Fig. 5b and d). The presence of gold nanosphere (Fig. $5 \mathrm{a}$ and $\mathrm{c}$ ) in between $\mathrm{Au}$ NRs creates hotspots via near-field mechanism and produced enhanced CD response [39]. The interaction of these nanosystems with biomolecules for utilizing it as a biosensor is in chaos. The Au NPs were assembled into 3D tetrahedrons with DNA origami template. The CD spectra of the $3 \mathrm{D}$ tetrahedrons exhibit bisignate signatures. The $\mathrm{CD}$ signal depends on the size of the Au NPs as well as the interparticle distance (Fig. 5). For same-sized Au NPs (20 nm), the intensity of $\mathrm{CD}$ signal continually increases with decrease in interparticle distance and decreases with decrease in size of Au NPs (13 nm) [40].

The arrangement of four identical Au NPs into 3D asymmetry tetramer using DNA origami template exhibiting 3D chirality [41]. Modular assembly of spherical Au NPs, Au NRs with definite numbers and heterogeneous nanostructures were fabricated with sample DNA origami template [42]. The DNA barrel scaffold induces the assembly of leftor right-handed tethers for the building-up of the chiral plasmonic Au NPs. The scalability of the DNA barrel structure into nanowire, followed by the deposition of Au NPs, could be initiated as chiroplasmonic wave-guides [43]. A dynamic $\mathrm{Au}$ NP plasmonic chiral nanostructures assembled using the DNA origami method in which the DNA alignment process gives several-fold increased CD signal[44].

The Au NR-based chiral plasmonic-excitonic systems were assembled on DNA template. The nanosystem shows Rabi splitting and anticrossing behaviour in the $\mathrm{CD}$ spectra. Rabi splitting occurs whenever the plasmon and exciton resonance energies are the same. The energy difference between symmetric mode and excitons is known as Rabi splitting. The splitting increases with an increase in the extent of coupling between excitonic molecule and chiral plasmonic nanostructures. The plasmon-like branch and exciton-like branch show transformations until the $\mathrm{CD}$ 

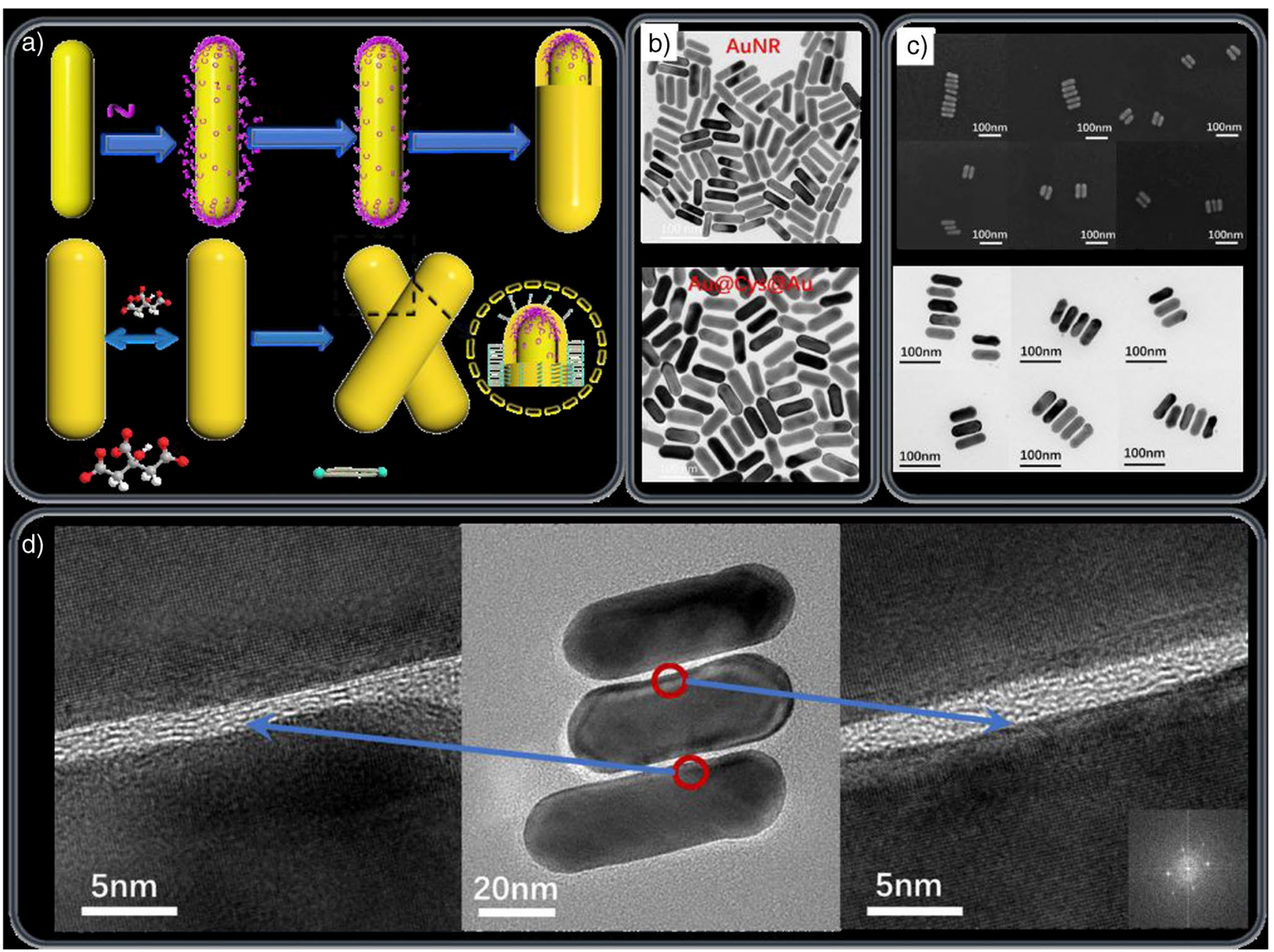

Fig. 4 a Schematic diagram of embedding sergeants in the interface region of $\mathrm{Au} \mathrm{NR}$ core and $\mathrm{Au}$ shell (upper panel). A chiral SS dimer is used to demonstrate the formation of chiral plasmonic assemblies (lower panel). The surfactant layer (soldiers) on the nanorod surface is omitted for clarity. b Representative TEM images of Au NR cores

resonances of the two branches become synchronized, thus exhibiting anticrossing property[45].

\section{Polymer-based chiral gold nanostructures}

The chiral self-assembled Au NRs with strong CD signal in the visible-near IR region has been successfully constructed using poly (2-hydroxyethyl methacrylate) (PIPEMA) and poly (methacrylate hydroyethyl-3-indolepropionate) (PHEMA). The pristine Au NRs do not induce any CD response. The intensity of bisignate longitudinal peak at $591 \mathrm{~nm}$ (positive peak) and $730 \mathrm{~nm}$ (negative peak) is stronger than the transverse peak at $528 \mathrm{~nm}$ (Fig. 6a). Here, the intensity of CD signal depends on the molecular weight of the polymers used as well as the $\mathrm{pH}$ values of the solution (Fig. 6a and d). The TEM and AuNR@Cys2@Au0.05 core-shell nanostructures. c SEM and TEM images of AuNR@Cys@Au SS oligomers. d A HRTEM image showing rod side facet link mode in a rod trimer. AA/Au3 + molar ratio=1.6. " Reproduced from Ref. 37 with permission from the Royal Society of Chemistry"

image and tilt angle $\left(0-60^{\circ}\right)$ TEM image show an L-handed side-by-side arrangement of Au NRs (Fig. 6b and c) [46].

The chiral arrangement of Au NPs using D- and L-helical pores in block copolymer films templates was fabricated. The arrangement of Au NPs in a porous poly(butadiene)-bpoly(ethylene oxide) film prepared using D-tartaric acid and L-tartaric acid with projection perpendicular and parallel to the cylinder axis are shown in Fig. 7. The TEM image consists of Au NPs with a pitch of average $40 \mathrm{~nm}$ (Fig. 7a and d) and 3D tomography further confirms the different helical chiral arrangements (Fig. 7c) [47]. 


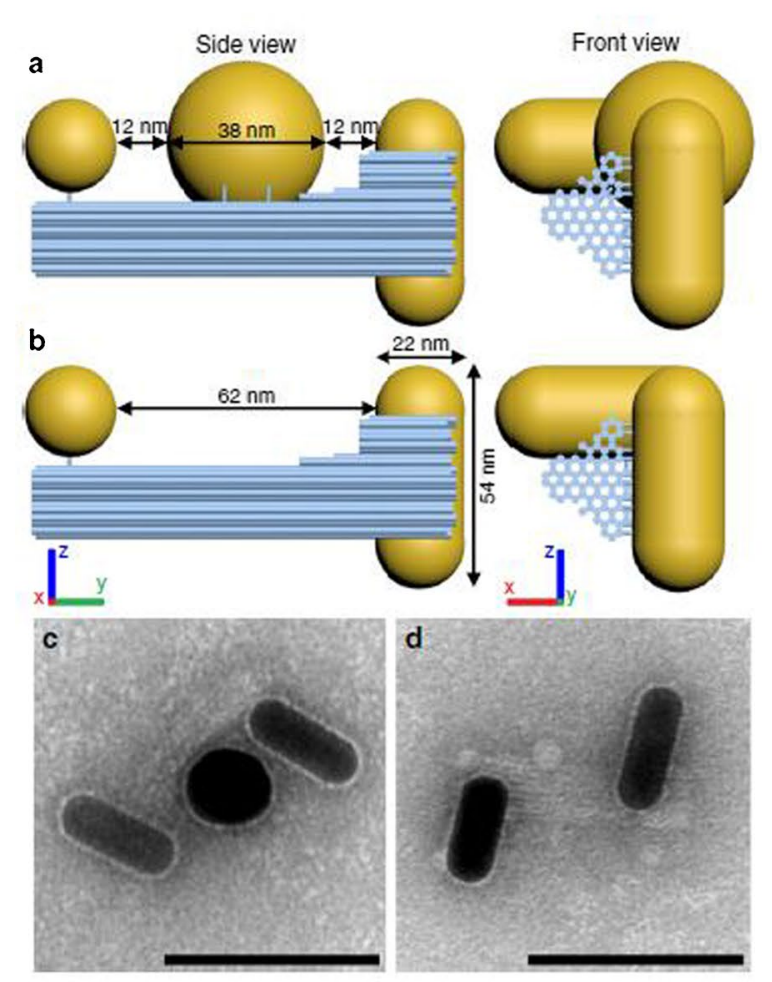

Fig. 5 Chiral plasmonic transmitter. a Side view and front view of DNA origami-nanoparticle assemblies in a nanorod-nanosphere $=$ nanorod (NR-NS-NR) arrangement and $\mathbf{b}$ a nanorod-voidnanorod (NR- -NR) arrangement. The nanorods and the nanosphere are mounted on a DNA origami structure (blue cylinders represent DNA helices) via thiolated DNA strands that are anchored to the origami structure. c Transmission electron micrograph of assemblies in the NR-NS-NR arrangement and $d$ in the NR- -NR arrangement. Scale bars: $100 \mathrm{~nm}$.CD spectra of the four Au NP tetrahedrons.

\section{Origin of chirality}

The plasmon-coupled circular dichroism (PCCD) emerges due to the dipole-dipole interaction between plasmonic nanoparticle and chiral adsorbate molecules. The PCCD was achieved in Au NRs-BSA aggregates. The CD arises in these aggregates by: (i) intrinsic structural chirality with enantiomeric excess of right-handed configuration, (ii) hot spots created between closely spaced Au NRs. The highangle annular dark-field scanning transmission electron microscopy (HAADF-STEM) imaging indicates that nonparallel dimer of Au NRs was circular differential scattering (CDS) active at $720 \mathrm{~nm}$ (Fig. 8A and B) whereas parallel dimer of Au NRs was CDS active at $618 \mathrm{~nm}$ (Fig. 8E and F). The tomographic reconstruction and simulations indicate that nonparallel dimer is chiral (Fig. 8C and D) and parallel dimer is achiral (Fig. 8G and $\mathrm{H}$ ) [48].

The gold nanostructures lock the chirality of circularly polarized light through the plasmonic field-driven asymmetric displacement of nanoparticles in dynamic ensemble
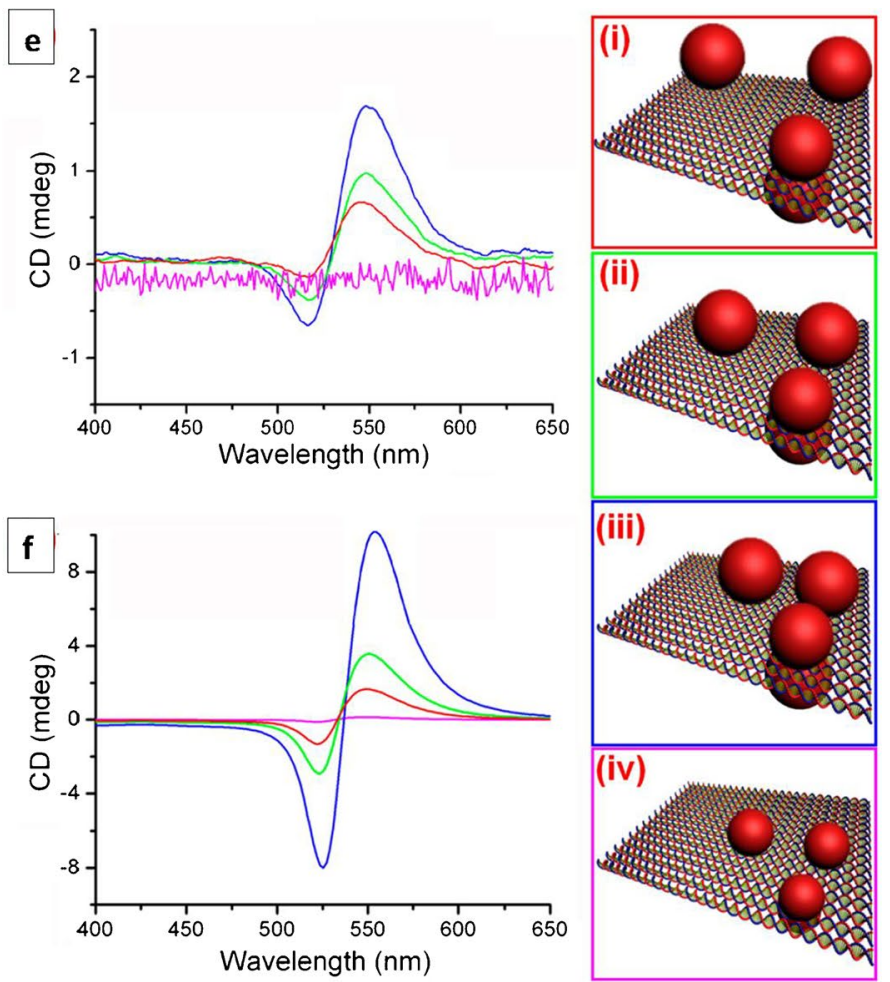

e Experimental data. f Calculated results. (i) 20-nm Au NPs with interparticle distance of $15 \mathrm{~nm}$ (red curve in e and f), (ii) 20-nm Au NPs with interparticle distance of $10 \mathrm{~nm}$ (green curve in e and f), (iii) $20-\mathrm{nm} \mathrm{Au} \mathrm{NPs} \mathrm{with} \mathrm{interparticle} \mathrm{distance} \mathrm{of} 5 \mathrm{~nm}$ (blue curve in e and f), (iv) 13-nm Au NPs with interparticle distance of $10 \mathrm{~nm}$ (magenta curve in e and $\mathbf{f}$ ). $\mathbf{a}, \mathbf{b}, \mathbf{c}$ and $\mathbf{d}$ Reprinted with permission from ref. 39. Copyright (2021) Springer Nature". e and f "Reprinted with permission from Ref. 40. Copyright (2014) American Chemical Society"

followed by particle-to-particle growth [49]. Chiral gold nanostructures were synthesized using L-cysteine and D-cysteine as capping agents [50]. The L-cysteine-encoded gold nanoparticle absorbs right-handed/clockwise circularly polarized light (CPL) at $534 \mathrm{~nm}$ and left-handed/anticlockwise CPL at $638 \mathrm{~nm}$. In the CD spectrum of D-cysteineencoded Au NPs, an inverted response at the same peak position is obtained (Fig. 9a). The 150-nm-sized rhombic dodecahedron Au NPs have 60-nm-sized edge with bent at an angle of $+\phi$, while for D-cysteine the same sized nanoparticle has edge with bent at an angle of $-\phi$ (Fig. 9b and c) [51]. The chiro-optical activity of amino acid and peptide directed synthesized gold nanoparticle depends on edge length, gap width, gap angle, growth rate, curvature and nature of additives [50]. The origin of chirality in $\mathrm{Ag}_{24} \mathrm{Au}_{1}(\mathrm{R} / \mathrm{S}-\mathrm{BINAS})_{\mathrm{x}}(\mathrm{DMBT})_{18-2 \mathrm{x}}$, $\mathrm{x}$ ranges from 1 to 7 , (DMBT $=2,4$-dimethylbenzenethiolate, BINAS $=$ R/S-1, 1 '[binaphthalene]-2,2'-dithiol) is due to the transitions within $\mathrm{Ag}_{24} \mathrm{Au}_{1} \mathrm{~S}_{18}$ framework and conjugation effects within the aromatic system of the ligand[52]. 

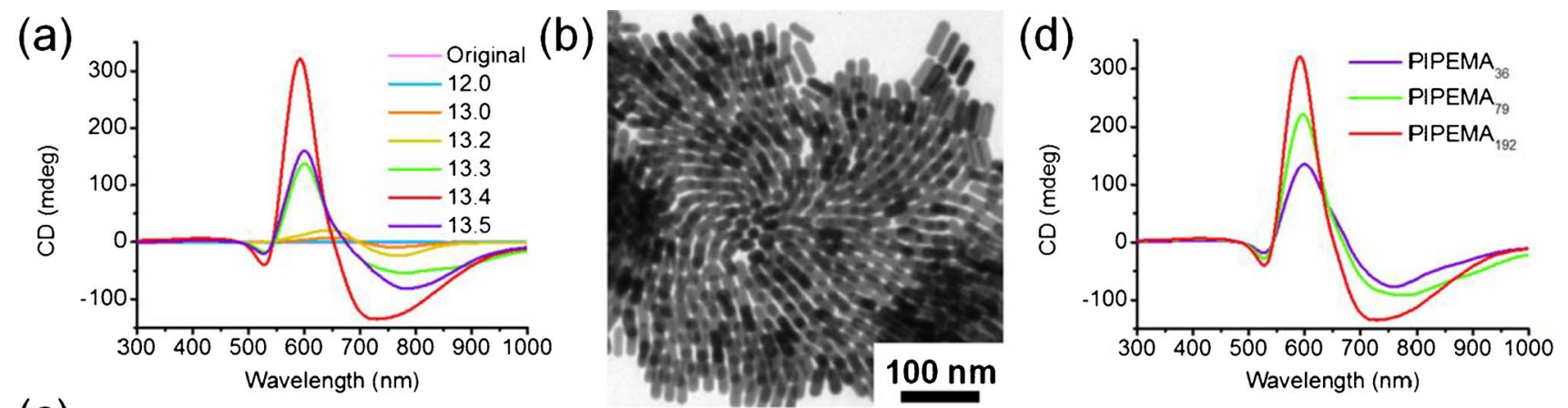

(c)
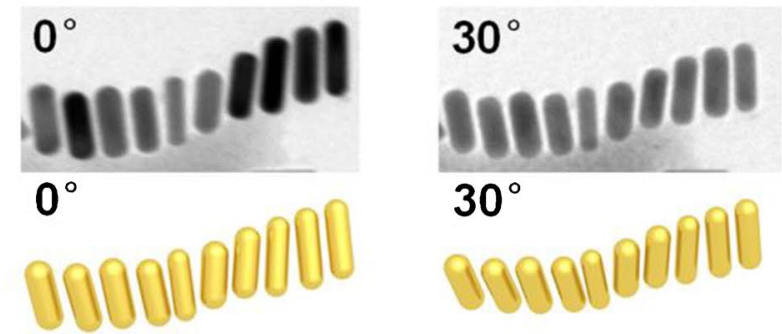

$60^{\circ}$

Anemede $100 \mathrm{~nm}$

$60^{\circ}$

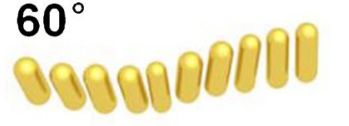

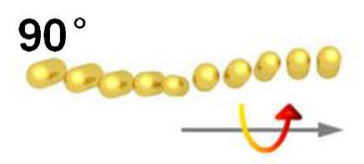

Fig. 6 a CD spectra of the original GNRs and the assemblies induced by PIPEMA192 under different $\mathrm{pH}$ values. The numbers in the label correspond to the $\mathrm{pH}$ value in the solution. b Representative TEM image of the assembled nanostructures at $\mathrm{pH}$ 13.4. c Top: tilt angle TEM images of a representative assembled structure, bottom: the cor- responding 3D model structures. The numbers in the label correspond to the title angle. d CD spectra of the GNR assemblies induced by PIPEMA with different molecular weights. "Reprinted with permission from Ref. 46. Copyright (2019) American Chemical Society"
Fig. 7 TEM image of chiral arrangement of Au NPs in a porous PBdEO film prepared using D-TA with projection a perpendicular to the cylinder axis and $\mathbf{b}$ parallel to the cylinder axis. The doping amount of TA in the parent BCP film is 16 wt $\%$. c 3D tomography of chiral arrangement of Au NPs in a porous $\mathrm{PBdEO}$ film using D-TA. d TEM image of chiral arrangement of Au NPs in a porous PBdEO film prepared using L-TA with projection perpendicular to the cylinder axis. "Reprinted with permission from Ref. 47. Copyright (2017) American Chemical Society"
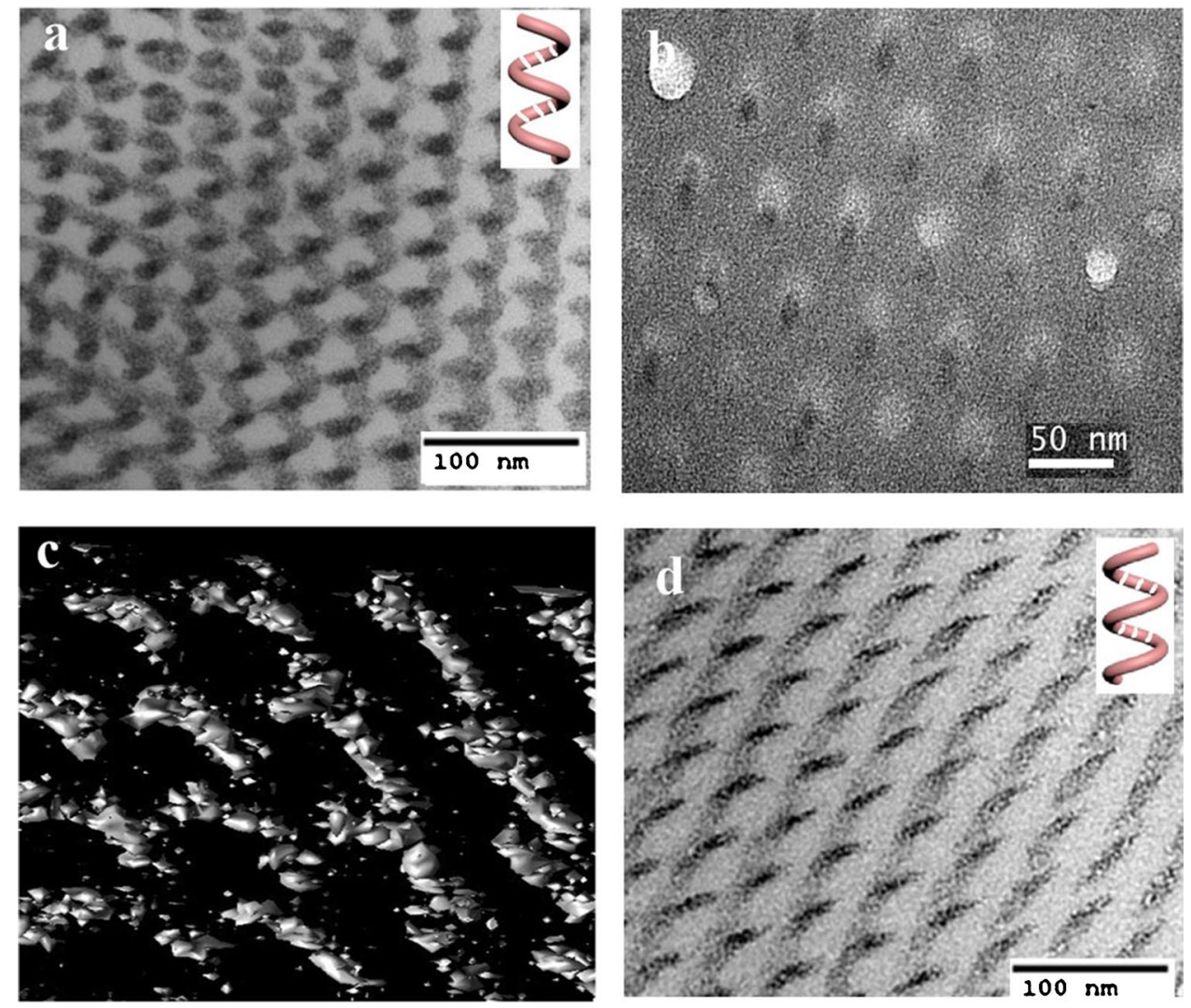

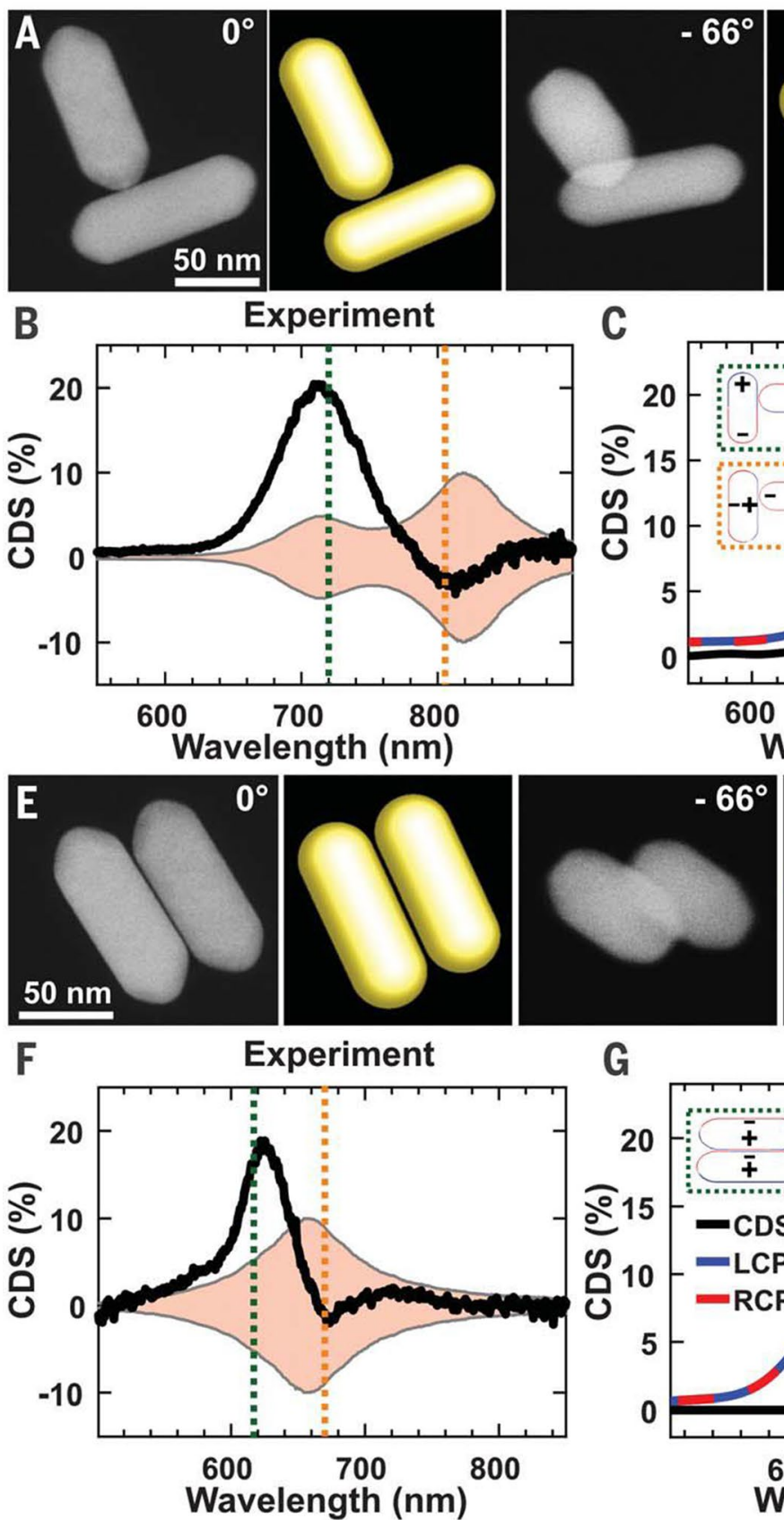

G

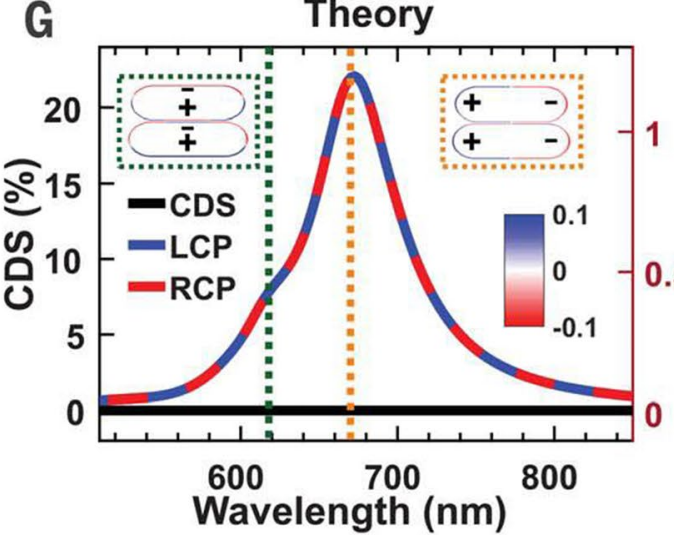

C

Theory

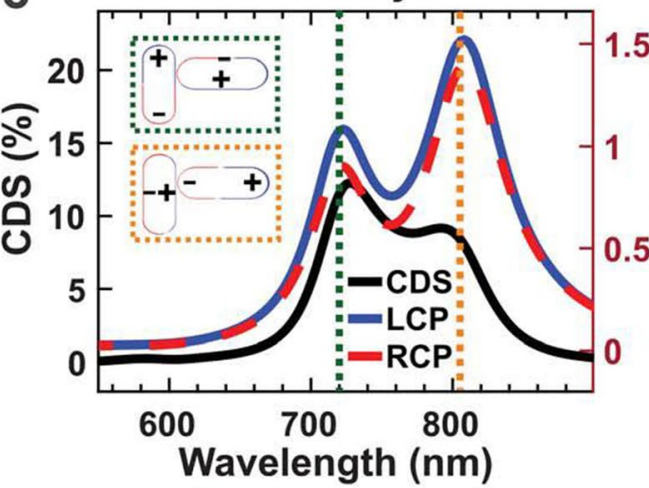

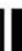

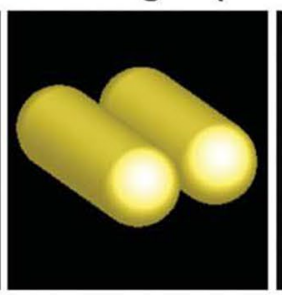

Theory
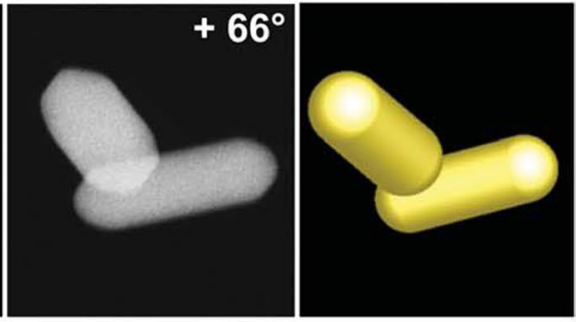

D

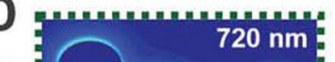

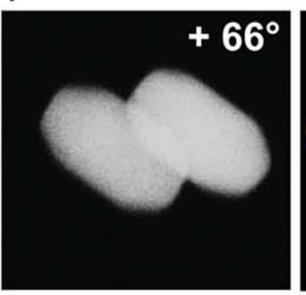

H

H
Fig. 8 CDS-active Au NR dimer-BSA complexes with chiral and achiral configurations. A and $\mathbf{E}$ HAADF-STEM tilt-series images of a chiral dimer (A) and an achiral dimer (E) and the corresponding geometric models extracted from the tomographic reconstructions. $\mathbf{B}$ and $\mathbf{F}$ Experimental single-particle CDS spectra of the chiral dimer (B) from (A) and the achiral dimer (F) from (E). The experimental spectra are shown with a pink envelope that represents the experimental error. $\mathbf{C}$ and $\mathbf{G}$ Simulated scattering spectra of the chiral dimer (C) and achiral dimer $(\mathrm{G})$ for incident LCP and RCP light and the corresponding CDS spectra. The insets in (C) show the charge plots calculated at 720 and $805 \mathrm{~nm}$. The insets in (G) show the charge plots calculated at 618 and $670 \mathrm{~nm}$. The charge plots in $\mathbf{C}$ and $\mathrm{G}$ share the same scale bar. The dashed lines in $\mathbf{C}$ and $\mathbf{D}$ refer to the plasmon modes at 720 (green) and $805 \mathrm{~nm}$ (orange). The dashed lines in $\mathbf{F}$ and $\mathbf{G}$ refer to the plasmon modes at 618 (green) and $670 \mathrm{~nm}$ (orange). $\mathbf{D}$ and $\mathbf{H}$ Cross-sectional views of calculated near-field enhancements $\left(\mathrm{lE} / \mathrm{E}_{0} 1^{2}\right)$ for the chiral dimer (D) at 720 and $805 \mathrm{~nm}$ and the achiral dimer $(\mathrm{H})$ at 618 and $670 \mathrm{~nm}$. These results demonstrate that although the dimer in $(\mathrm{E})$ to $(\mathrm{H})$ is not chiral on the basis of its geometry, CDS is observed and must originate from chiral BSA molecules located in inter-NR hotspots. a.u., arbitrary units; E, electric field; $\mathrm{k}$, incident wave vector. " Reproduced with permission from Ref. 48. Copy right 2019, The American Association for the Advancement of Science" 


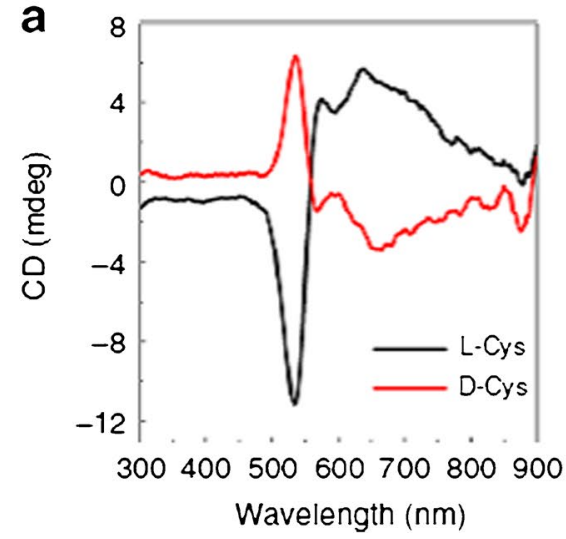

Fig. 9 Opposite handedness of chiral nanoparticles depending on the cysteine enantiomer. a Circular dichroism (CD) spectrum of nanoparticles synthesized using L-cysteine (L-Cys, black) and D-cysteine (D-Cys, red). SEM images of resultant chiral nanoparticles obtained using L-Cys (b) and D-Cys (c). Schematic models and magnified

The three main spectroscopic techniques for the characterization of chirality in materials include optical rotatory dispersion (ORD), CD and Raman optical activity (ROA). $\mathrm{CD}$ is related to the imaginary part of the chirality parameter " $k$." It depends on ellipticity, which is a function of the proportionate difference in transmission. The ORD is related to the real part of chirality parameter. It is expressed as the unequal rotation for plane polarized light of different wavelengths [53]. Optical activity is the ability of a molecule to rotate the plane polarized light [54].

\section{Intrinsic plasmonic chirality}

In 3D chiral plasmonic nanomaterials, the circular dichroism effect occurs due to the interaction of (i) electric dipole and magnetic dipole and (ii) electric and magnetic dipoles with dipole-quadruple responses of the system. A 3D chiral configuration resulted from the wave vector, electric dipole and magnetic dipole. The interplay of electric and magnetic dipole results in an imaginary part of mixed electric and magnetic polarizability. This mixed polarizability is a dot product of both these dipoles. The helical arrangement of Au NPs bring about coupled plasmon waves disseminating along a helical path associated with absorption of these waves with respect to the handedness of the plasmonic nanohelices [55].

\section{Extrinsic plasmonic chirality}

Extrinsic plasmonic chirality resulted from the mutual alignment of the 2-D metamaterial and the direction of
SEM images are shown in the inset. Auxiliary lines indicating oppositely bent edges at an angle of $+\phi$ for L-Cys and $-\phi$ for D-Cys are illustrated in the SEM images. Scale bar, $100 \mathrm{~nm}$. Reproduced with permission from Ref. 51. Copyright $\odot 2020$, Springer Nature

wave propagation [54]. Extrinsic chirality of 3D-chiral metamaterials occurs by the oblique incidence onto metasurfaces, if it lacks line of mirror symmetry in the direction of plane of incidence $[56,57]$. The extrinsic chirality via asymmetric transmission due to oblique incidence can occur in square arrays of plasmonic nanospheres, semiconductor quantum dots, planar metamaterials and lossy double-periodic gratings [58]. The extrinsic chirality through photoluminescence emission takes place by breaking the mirror symmetry in the presence of asymmetric $\mathrm{Au}$ coating [59]. The oblique excitation/illumination of a 2D achiral plasmonic nanostructure at a particular incident angle exhibits a non-zero plasmonic CD response through extrinsic chirality [60]. Such extrinsic chirality happens by the strong interplay of electric and magnetic dipoles leading the way to mixed polarizability. Under oblique incidence, symmetry breaking and the projection of the oscillating magnetic moment on the plane perpendicular to the wave propagation induced by CPL are nonzero [61].

The huge extrinsic CD of a plasmonic splitting rectangle ring originates due to the generation of circular current by acting as a magneton is verified using the FEM simulation. In analytical model, the CD of meta-molecule from left and right circularly polarized light occurs due to mixed electric and magnetic polarizability [62]. The $\mathrm{Au}-\mathrm{Ag}$ heterodimer behaves like a plasmonic magneton. It possesses multipolar Fano-resonances and powerful CD effect. Both Fano-resonance profile and CD signal depends on size, gap and shape of nanorice heterodimer; the CD spectra are sensitive to ambient medium in visible and near IR regions. In addition to it, the $\mathrm{CD}$ effect varies constructively with Fano-asymmetry factor [63]. 


\section{Chiral fields in dipoles under external excitation}

The generation of strong chiral fields in the vicinity of a dipole dimer is illustrated in Fig. 10. An enhanced and uniform chiral field was formed in the gap between the two dipoles placed in such a way that two lobes with the same chirality get overlapped. In the corners of Au block dimers, strong field enhancement occurs, resulting in drastic chiral fields. The chiral metal nanostructure and non-chiral structure with CPL illumination, the chiral field is nonuniform and the enhancement changes between positive and negative at different wavelength. In the case of helical structures excited with linearly polarized light, the chiral field is uniform. Here, the enhancement factors are either negative or positive [64].

\section{Discrete dipole approximation}

In multipoles, thousands of dipoles are considered as individual particles. The plasmonic $\mathrm{CD}$ response of such multipoles is taken by averaging the variance in extinction cross sections, Q, of left circularly polarized from right circularly polarized plane waves by considering different orientation of the helical superstructures $C D=<\mathrm{Q}_{\mathrm{IL}}-\mathrm{Q}_{\mathrm{IR}}>\Omega$, where $Q_{I L}$ and $Q_{I R}$ indicates extinction cross section of left circularly polarized plane waves, and right circularly polarized plane waves, respectively, $\Omega$ represents the set
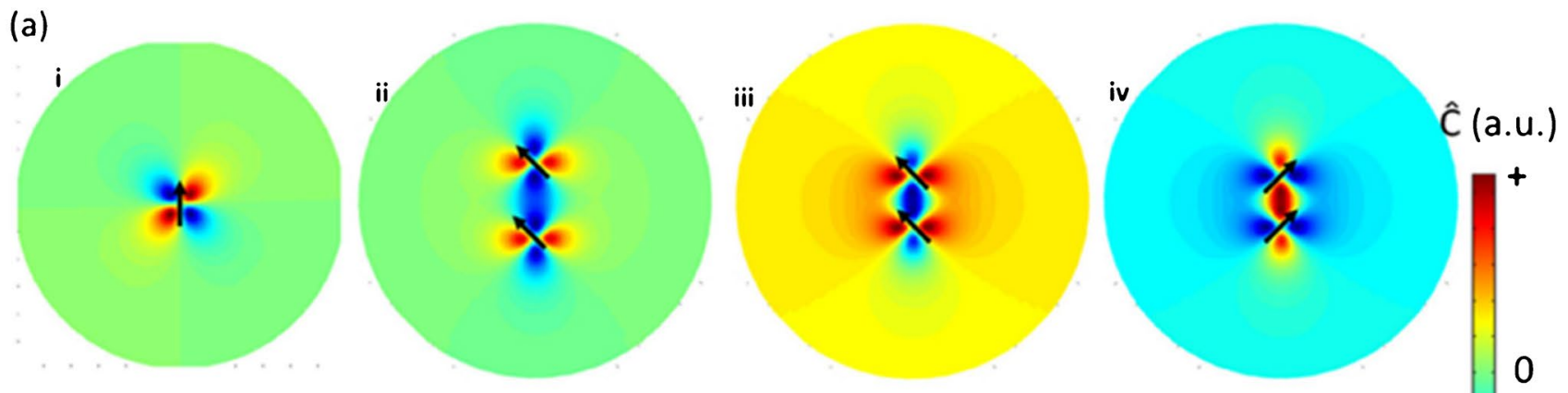

(b)
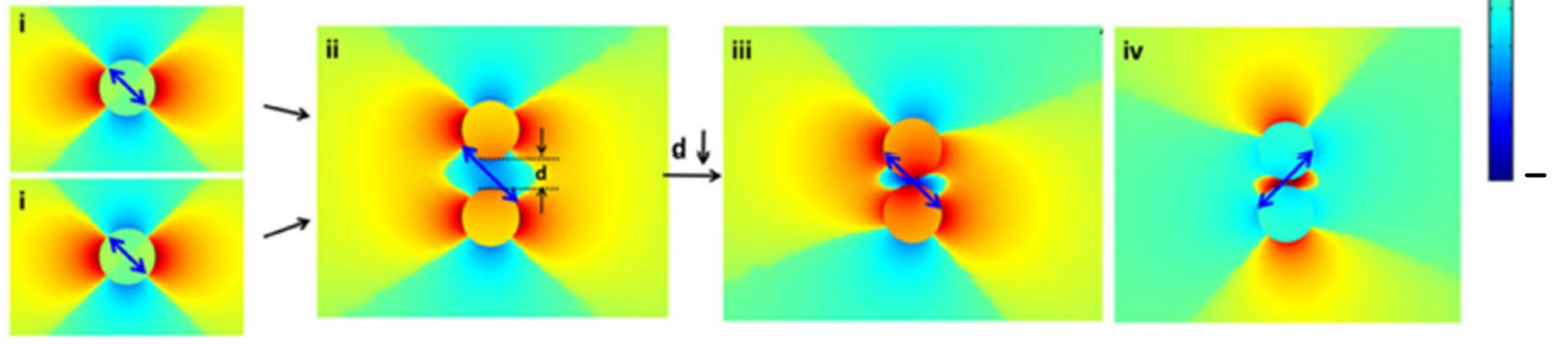

(c)
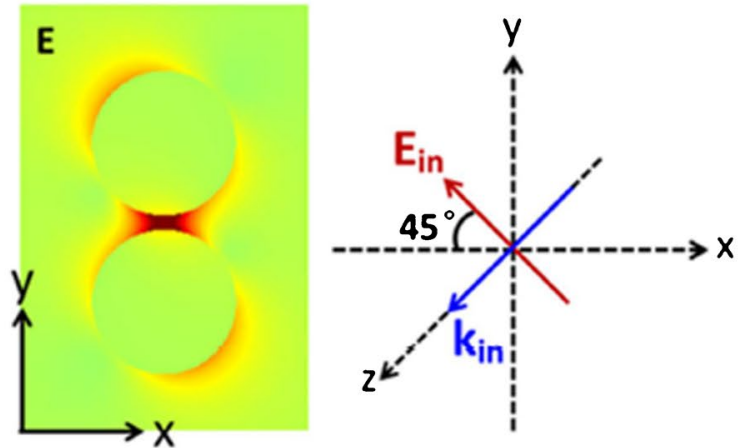

Fig. 10 Formation schematic of enhanced chiral near-fields with uniform optical chirality in the gap of a coupled point dipole dimer (a) and Au spherical nanoparticle (10-nm diameter) dimer (b). a Analytically calculated chiral near-field distributions of (i) one dipole, (ii) two dipoles with a large gap $d$ of $0.06 \lambda$, and (iii-iv) two dipoles with a small gap d of $0.04 \lambda$. Black arrows show the dipole momentum. Signs of "+" and "- " in the scale bar indicate the field is left- and right-handed, respectively, which applies to all figures in the follow- (d)

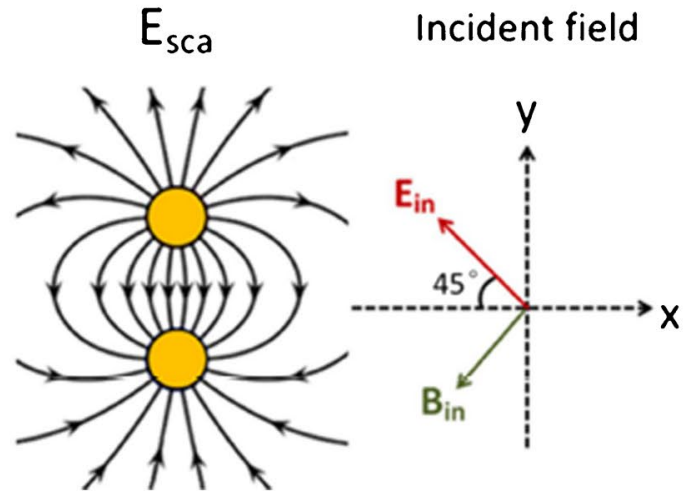

ing. b Numerically calculated chiral near-field distributions of (i) one sphere, (ii) two spheres with a large gap d of $10 \mathrm{~nm}$ and (iii-iv) two dipoles with a small gap d of $2 \mathrm{~nm}$. c Corresponding electric field distribution of the case (b)-iii. The right coordinates give incident polarization and direction. $\mathbf{d}$ Schematic of the directions of incident fields and scattered fields by a dipole dimer. Reproduced with permission from Ref. 64. Copyright (C) 2015, Springer Nature 
of orientations of the helical superstructure with respect to the incident wave-vector [65]. The total CD signal in a system comprising of a nanocrystal and a chiral molecule is $\mathrm{CD}_{\mathrm{NP} \text {-molecule }}=\mathrm{CD}_{\mathrm{NP}}+\mathrm{CD}_{\text {molecule, }}$, Where $\mathrm{CD}_{\mathrm{NP}}=<\mathrm{Q}_{\mathrm{NP},+}$ $-\mathrm{Q}_{\text {molecule }},->\Omega$, where $\mathrm{Q}_{+(-)}$are the rate of absorption corresponding to two incident electromagnetic waves. The molecule produces a strong Fano effect in the CD spectra. This $\mathrm{CD}$ band of molecule appears at the plasmon frequency. The plasmonic nanoparticles like gold and silver nanoparticle exhibit an absorption enhancement with small separation between molecules and nanoparticle [66].

\section{Optical coupling between gold nanorods and chiral surfactants}

The Au NRs coated with chiral mesoporous silica shells (CMS) manifest strong CD signals in the visible light and NIR region (Fig. 11a). The CD activity is due to the optical coupling between Au NRs and the chiral cysteine molecules docked in the CMS shells. The small CD signals at $520 \mathrm{~nm}$ correspond to the feeble transverse LSPR while strong CD signal ranging from 500 to $900 \mathrm{~nm}$ are attributed to the intense longitudinal LSPR (Fig. 11b). In the absence of chiral mesoporous silica, the mixing of Au NRs with pure chiral molecules lacks CD signal in the visible region, while that in the UV region remains. With an increase in aspect ratio of $\mathrm{Au}$ NRs, the plasmonic peak around $700 \mathrm{~nm}$ gets red-shifted. It indicates that the origin of plasmon-induced chiral property is due to the strong near-field coupling between chiral molecules within the mesoporous shell and Au NRs.[67].

\section{Chirality induced by preferred handedness of the polymer chain}

Using the chain transfer polymerization of PIPEMA and PHEMA leads to the self-assembly of Au NRs, with $\mathrm{CD}$ response at Vis-NIR region. The CD spectra of the nanoassembly give a maximum intensity peak at $\mathrm{pH}$ of 13.4. The origin of chirality of Au NRs is controlled by the preferred handedness of the chiral polymers owing to the syndiotacticity and steric hindrance of the side groups of the polymer chain. Moreover, the intensity of the CD signals is controlled by the molecular weight of the polymers [46].

\section{Born-Kuhn model}

The plasmonic form of the Born-Kuhn model can be well illustrated as two identical corner-stacked, vertically aligned nanorods having an angle of $90^{\circ}$ between them. These orthogonal nanorods are equivalent to chiral plasmonic dimer. In coupled oscillator model the excitation of a mode depending on symmetry of light corresponds to antisymmetric case. Hybridization model gives idea about sign and spectral position of the resulting optical modes. The plasmonic Born-Kuhn model demonstrates the tuning of vertical inter-rod distance, which can open on to selective excitation of bonding and antibonding plasmonic chiral modes [68]. The Born-Kuhn-type plasmonic analog produced with 3D gold chiral metasurfaces was used for the analysis of third-harmonic generation circular dichroism (THG-CD). The THG-CD spectra consist of broader peak and dip than that of linear CD spectra. In addition to this, the nonlinear $\mathrm{CD}$ peaks is almost zero at the peak position of linear $\mathrm{CD}$ spectra [69].

\section{Tuning of chirality of Au NPs}

The chiroptical activity of chiral Au NPs can be tuned by the peptide sequence. The sequence engineering of A3 goldbinding peptide (AYSSGAPPMPPF) can be used for optimizing chiroptical properties of chiral helical Au NPs. The similar CD spectra show that all peptides have polyproline II secondary structure (Fig. 12). The position of methionine
Fig. 11 a CD and $\mathbf{b} \mathrm{UV}-\mathrm{Vis}$ spectra of the GNR@CMS NPs templated by three types of different chiral surfactants: C16-LPhe (black); C16-D-Phe (red); C16-DL-Phe (blue). "Reprinted with permission from Ref. 67. Copyright (2013) American Chemical Society"

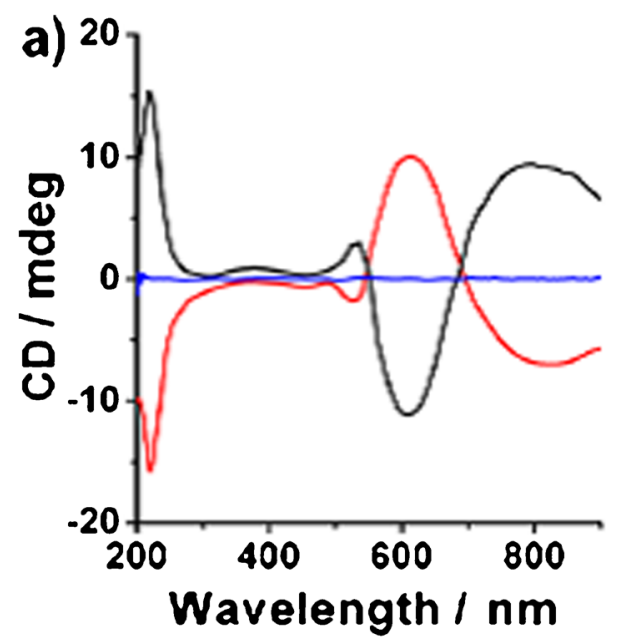


Fig. 12 Secondary structure analysis of PEPAuM,11 and PEPAuM-ox, 11. a CD measurements indicate that both PEPAu M,11 and PEPAu M-ox,11 exhibit predominantly PPII secondary conformations in solution. b Structural similarity between PEPAu M,11 (blue) and PEPAu M-ox,11 (red) sequences gathered via theoretical cross-peptide analysis "Reprinted with permission from Ref. 70. Copyright (2019) American Chemical Society"
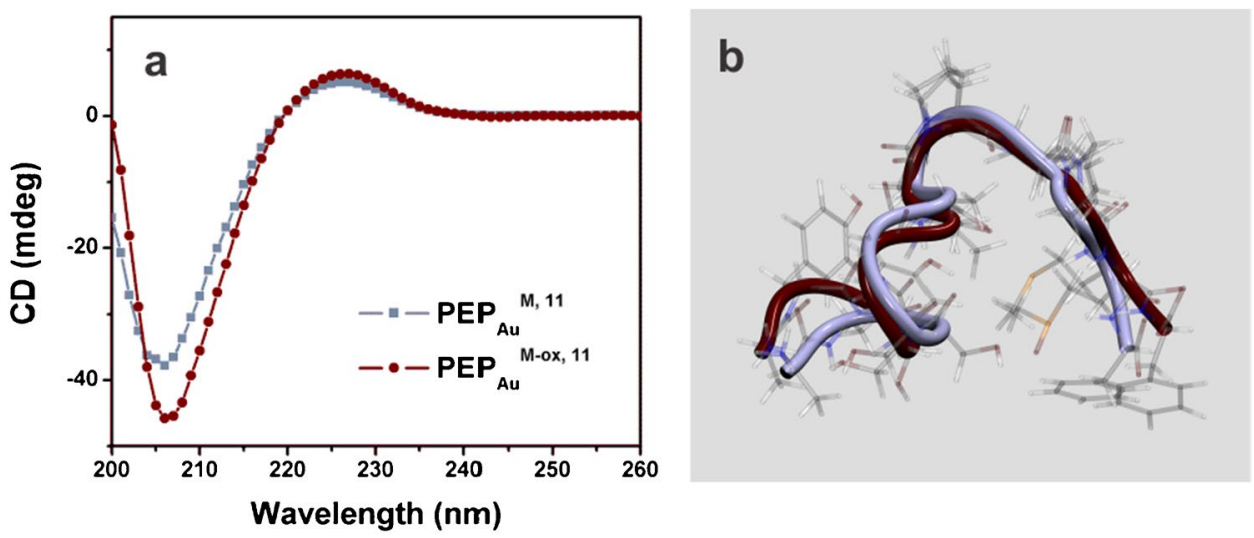

(M) or methionine sulphoxide (M-ox) within the peptide sequence controls the aspect ratio of Au NPs. The nature of the peptides determines the formation of spherical, oblong or rod-shaped Au NPs. The shift in the M-ox position of peptides gives large-sized oblong Au NPs with high intensity of CD peaks [70]. A series of Au NRs capped with axially chiral binaphthyl thiols and its dispersion in nematic liquid crystals 5CB have been synthesized. The enhancement of chirality by a related series of polyhedral Au NRs can be employed to identify chirality transfer. The nematic liquid crystal phase reflects the variation in chiral induction strength and facilitated cooperative effects with helically arranged chiral objects [71]. In a polar protic solvent, chiral gold nanoclusters (Au NCs) undergo self-aggregation into supraparticles, while in polar aprotic solvent, self-aggregation does not occur. The degree of self-aggregation increases with an increase in polarity of polar protic solvent, which induces amplification and chirality of supraparticles [72].

The Au NPs functionalized with a highly sensitive and conformational responsive enantiomeric pairs of axially chiral binaphthyl ligands were employed to induce the chirality transfer from nanoscale solid particle to a bulk achiral liquid crystal phase. The Au NP-conjugated binaphthyl ligands were used for analysing the dependent factors of chirality transfer such as size of Au NPs, chiral correlation length, the local number, dihedral angle and density of chiral binaphthyl ligands [73]. A coordinating thermally sensitive organogelator template controls the chirality in the in situ synthesis of hybrid Au NPs. The sol form of the template induces coordination between $\mathrm{Au}(\mathrm{III})$ and azobenzene moiety results in chirality inversion, whereas the gel form of the template cannot coordinate with $\mathrm{HAuCl}_{4}$ so that original chirality is maintained [74]. The chiral poly(fluorene-altbenzothiadiazole) doped with helically ordered Au NPs via dipole-dipole interaction leads to plasmonic enhancement of optical chirality parameter (Fig. 12) [75]. The circular differential Mie scattering (CDMS) of Au NPs is linearly proportional to the resonance strength and chirality parameter $(k)$ of the molecules [76]. The three chiral Au NR-chromophore hybrid structures were constructed by self-assembling achiral cyanine dye K21J-type aggregates, cyanine dye (cy3) and cyanine dye (cy5) with Au NRs with the aid of DNA templates. The chirality of the hybrid complexes depends on the spectral overlap between the SPR and chiral collective molecular excitations [33].

\section{Mechanism of CD effect in chiral plasmonic gold nanostructures}

The mechanism of CD effect in chiral plasmonic gold nanostructure is based on coulomb interaction, plasmon-induced mechanism and dipole-dipole interaction. The coulomb interaction between molecule and nanoparticle creates an angle separation between electric and magnetic dipole of the molecule (Scheme 1a and b). The interaction of nanoparticle creates a CD effect via lowering the symmetry of the molecule. The dipole of the molecule induces a chiral current inside the nanoparticle. It results in a Fano effect in the CD spectra. The CD spectra due to the interaction of a molecule and nanoparticle in the presence of an electric field comprises of (i) incident field, (ii) field induced by nanoparticle and (iii) field created by the molecule. The asymmetric shapes of CD bands of a nanoassembly are called Fano-type effect. In Scheme 1a, the electric and magnetic dipole $\vec{\mu}_{12}$ and $\vec{m}_{21}$, respectively, are not perpendicular to each other, thereby resulting in CD signal. Here, $R$ is centre to centre separation and $\Delta$ is the distance between the centre of the molecule and nanoparticle surface [66].

The chiral adsorbate on Au NPs interacts with the electronic states of the nanoparticle and generates a CD band at the plasmon wavelength (Scheme 1c and d) [77]. This type of mechanism is applied to the nanosystem in which the absorption peak of the chiral molecule is far away from plasmonic band of the nanoparticle. Here, the CD effect is induced due to the modification of the surface state of the nanoparticle via the adsorption of the chiral molecule [66]. 
Scheme 1 Schematic representation showing the mechanism of origin of CD effect. a The direction of magnetic vector and electric vector. b The coulomb interaction between energy states of dye molecule and the Au NPs. $\mathbf{c}$ and $\mathbf{d}$ The change in photo-induced CD signal with variation in the direction of electric vector of molecule. e Non-parallel interaction of chiral molecule (molecular plane) with the electric dipole of Au NPs and $\mathbf{f}$ the parallel interaction of chiral molecule (molecular plane) with the electric vector of Au NPs

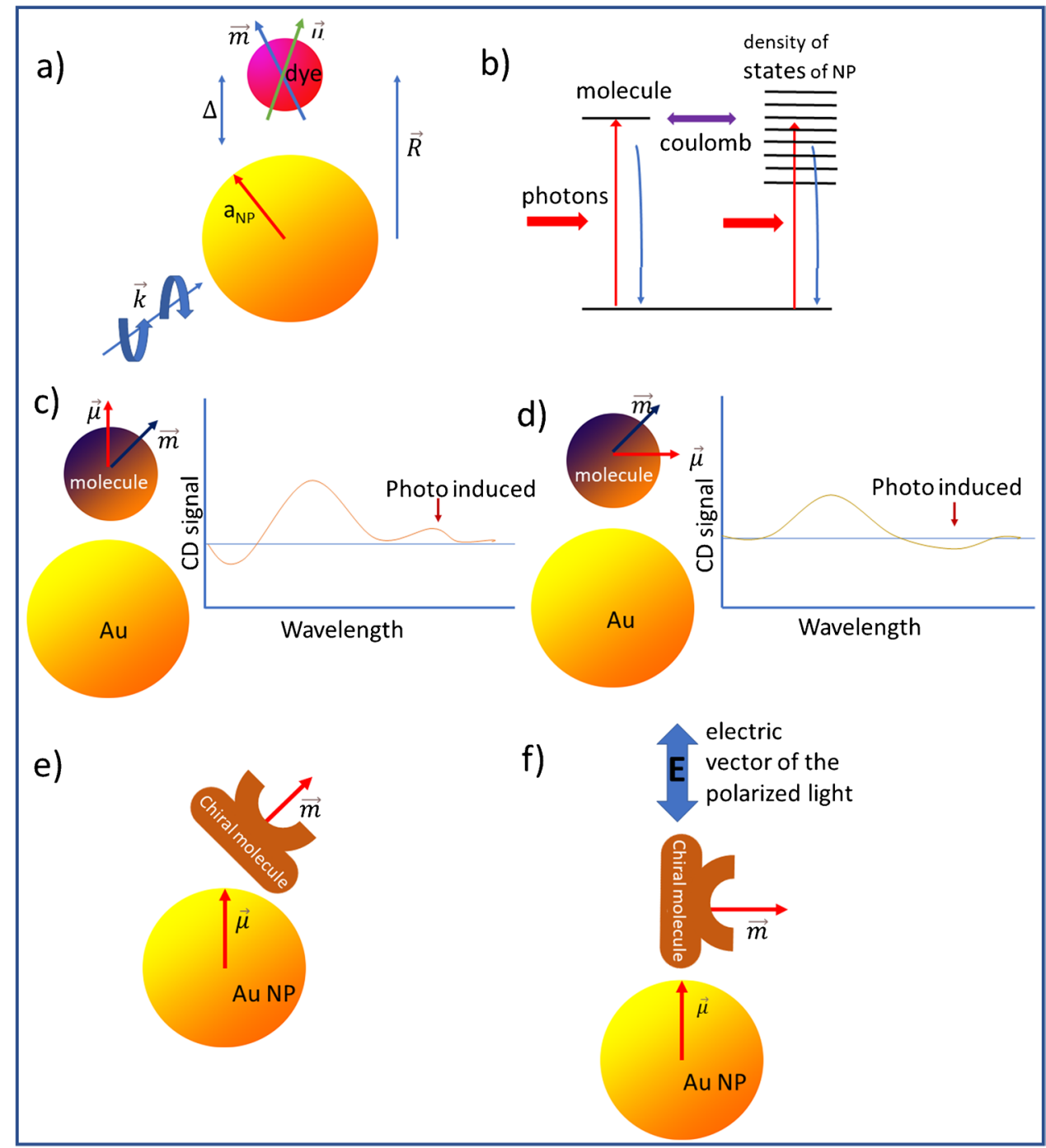

In hybrid materials such as azo-compounds and Au NPs in polymer films, the two components of dipole vectors can freely rotate in a direction perpendicular to the direction of propagation of light. The CPL can induce supramolecular chirality with one vector on the surface of Au NPs and the second dipole vector is fixed within the Au NPs (Scheme 1e and $\mathrm{f}$ ). Here, the initial weak adsorption of chiral species on the surface of Au NPs limited the motion of the resulting complex (Scheme 1e) [78].

\section{Applications of chiral plasmonic gold nanostructures}

Chiral plasmonic gold nanostructures are established as a probe for a wide variety of applications including catalysis, biosensing and biodetection. Chiral, multipetal gold nanoflowers (Au NFs) were prepared by the reduction of $\mathrm{HAuCl}_{4}$ with the aid of core-satellite silver nanoparticle seeds. The $\mathrm{Hg}^{2+} / \mathrm{Au}$ NF combination catalyses the transformation of rhodamine $\mathrm{B}$ to rhodamine 110 and nitroarenes to aminoarene. The bright-field TEM images of Au NF look like mermaid flower (Fig. 13a and b). The HRTEM images consist of lattice planes with spacing $2.33 \AA$ corresponding to (111) plane of fcc Au NFs (Fig. 13c). The STEM-HAADF (scanning transmission electron microscopy/high-angle annular dark field) (Fig. 13d), energy-dispersive X-ray (EDX) (Fig. 13e), energy-filtered TEM (EFTEM) image (Fig. 13g), $\mathrm{CD}$ spectra and wide-angle XRD profile of Au NF prove the characteristic properties of gold. Relative thickness map of individual Au NF (Fig. 13f) is shown in Fig. 13h [79].

\section{Biosensing}

Chiral plasmonic gold nanostructures exhibiting CD were used for biosensing because they are sensitive to asymmetry in sample structure, quick easy method in obtaining measurements, shorter time scale, less amount of sample required 
Fig. 13 a and b BF-TEM images of Au NFs and a single Au NF, respectively, and c HRTEM image of a part of $\mathrm{Au}$ NF. d STEM-HAADF image. e EDX line profile. f Unfiltered image of a single Au NF. $\mathbf{g}$ EFTEM image of that Au NF. $\mathbf{h}$ Relative thickness map. i Line profile over the red rectangular box indicated in $\mathbf{h}$. $\mathbf{j}$ CD spectra of chiral Au NFs. $\mathbf{k}$ Wideangle XRD profile of Au NFs. "Reprinted with permission from Ref. 79. Copyright (2015) American Chemical Society"
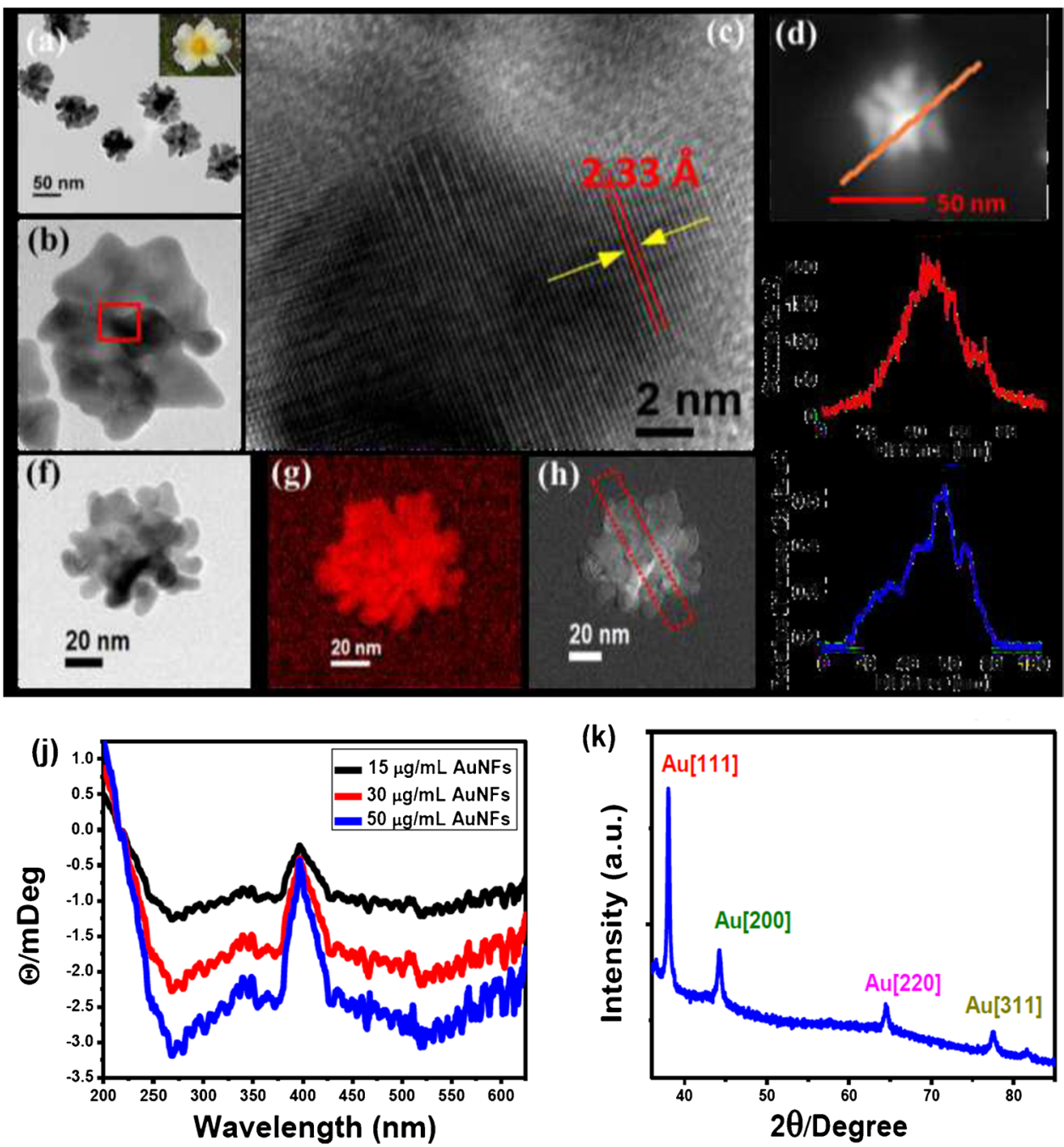

and recovery of sample is possible [80]. CD exploits the differential absorption of right- and left-handed plane polarized light sensitive to asymmetric in structure of molecules [81]. Recently, chiral plasmonic gold nanostructures offer new platform for highly sensitive detection strategies. Because of their biocompatibility and plasmonic properties, gold nanostructures can make sensible effect in the detection process.

Chiral plasmonic gold nanostructures can find the way towards various biosensing applications, including labelfree single-molecule/virus detection. The antibody-conjugated self-assembled superstructure of chiroplasmonic gold nanoparticles (CAu NPs) and CdTe NPs quantum dots (QDs) nanohybrids could detect picomolar concentrations of avian influenza $\mathrm{A}\left(\mathrm{H}_{5} \mathrm{~N}_{1}\right)$ virus. The CdTe NPs exhibit a particle size of $6.5 \mathrm{~nm}$ with strong emission wavelength at $710 \mathrm{~nm}$ and possibly could overlap with plasmonic states in the nanohybrids. Here, prolate, flower and urchin-shaped Au NPs having plasmonic peaks at $548 \mathrm{~nm}, 565 \mathrm{~nm}$ and $590 \mathrm{~nm}$, respectively, were chosen to make nanohybrids. The urchin-shaped Au NPs outperform all other Au NPs because of their broadened plasmonic peak and maximum extent of overlap with excitonic wavelength of CdTe NPs. The sensitivity of the nanohybrid-based bioassay could detect up to 1 pgmL $^{-1}$ of $\mathrm{H}_{5} \mathrm{~N}_{1}$ virus, which was 10 times more than that of bioassay based on bare urchin-like Au NPs. Moreover, chiroptical response is more sensitive as compared to visual colour response-based commercial kit, which could detect up to $1 \mathrm{ngmL}^{-1}$ (Table 1). The sensing performance was also extended to detect avian influenza A (H4N6) virus, coronavirus and fowl adenovirus. The concept of optical rotation activity and energy coupling characteristics due to chiral plasmon-exciton interactions change the chirality enhancing the sensitivity of chiral plasmonic nano sensors [82].

A chiral assembly of 16-mercaptohexadecanoic acid-functionalized Au NPs immobilized with tropomyosin (TROP) and anti-tropomyosin monoclonal (mAb) antibodies as trimer was developed for the sensing and quantification of shell fish allergen tropomyosin. In the nanosensor, Au NPs-TROP comparatively binds with $\mathrm{Au}$ NPs-mAb and free antigen. The AuNPs-TROP and Au 
Table 1 Summary of chiral plasmonic gold nanostructure-based assays for various detection targets

\begin{tabular}{|c|c|c|c|c|c|}
\hline Target & Nanomaterial & Real sample & Linear range & Detection limit & Ref \\
\hline $\begin{array}{l}\text { Avian influenza A H4N6 } \\
\text { virus }\end{array}$ & $\begin{array}{l}\text { Antibody conjugated } \\
\text { self-assembled gold nano } \\
\text { structure and CdTe NPs }\end{array}$ & Chicken blood & 100-0.01 HAU/50 $\mu \mathrm{L}$ & $0.0315 \mathrm{HAU} / 50 \mu \mathrm{L}$ & [82] \\
\hline $\begin{array}{l}\text { Fowl adenoviruses-9 } \\
\text { (FAdVs-9) }\end{array}$ & $\begin{array}{l}\text { Antibody conjugated } \\
\text { self-assembled gold nano } \\
\text { structure and CdTe NPs }\end{array}$ & Chicken blood & & $33.64 \mathrm{PFU} \mathrm{mL} \mathrm{m}^{-1}$ & [82] \\
\hline Bronchitis virus (IBV) & $\begin{array}{l}\text { Antibody conjugated } \\
\text { self-assembled gold nano } \\
\text { structure and CdTe NPs }\end{array}$ & Chicken blood & $10^{2}-10^{4} \mathrm{EID} / 50 \mu \mathrm{L}$ & $47.91 \mathrm{EID} / 50 \mu \mathrm{L}$ & [82] \\
\hline Tropomyosin & Chiral Au NP trimer & Shell fish & $0.1-15 \mathrm{ngmL}^{-1}$ & 21 pgmL-1 (S/N=3) & [83] \\
\hline DNA & Au heterodimers & N/A & $160 \mathrm{zM}-1.6 \mathrm{pM}$ & $17 \mathrm{zM}$ & [86] \\
\hline Prostate-specific antigen & $\begin{array}{l}\text { Functionalized Ag NP-Au } \\
\text { NP heterodimers }\end{array}$ & Serum & $1 \times 10^{-9}$ to $1 \times 10^{-6}$ & $5 \times 10^{-10} \mathrm{ng} / \mathrm{mL}$ & [87] \\
\hline MCLR & $\begin{array}{l}\text { Functionalized Ag NP-Au } \\
\text { NP heterodimers }\end{array}$ & Water from lake & 0.002 to $0.5 \mathrm{ng} / \mathrm{mL}$ & $0.0008 \mathrm{ng} / \mathrm{mL}$ & [87] \\
\hline miRNA & $\begin{array}{l}\text { Gold-upconversion nano- } \\
\text { particle }\end{array}$ & HeLa cells & $\begin{array}{l}0.073 \text { to } 43.65 \mathrm{fmol} / 10 \\
\mu \mathrm{g}_{\mathrm{RNA}}\end{array}$ & $0.03 \mathrm{fmol} / 10 \mu \mathrm{g}_{\mathrm{RNA}}$ & [32] \\
\hline ATP & $\begin{array}{l}\text { Gold nanoparticle heter- } \\
\text { odimers }\end{array}$ & live cell & 1.5 to $4.2 \mathrm{mM}$ & $0.2 \mathrm{mM}$ & [89] \\
\hline $\mathrm{Zn}^{2+}$ & $\begin{array}{l}\text { Au@AuAg yolk-shell } \\
\text { nanorods }\end{array}$ & human SH-SY5Y cells & $\begin{array}{r}76.6 \times 10^{-6} \mathrm{M} / 10^{6} \text { cells to } \\
211.5 \times 10^{-6} \mathrm{M} / 10^{6} \text { cells }\end{array}$ & $\begin{array}{l}38.7 \pm 0.3 \times 10^{-6} \mathrm{M} / 10^{6} \\
\quad \text { cells }\end{array}$ & [90] \\
\hline
\end{tabular}

NPs-mAb could form aggregates of different oligomers and contributes to the increase in CD intensity. The biosensor was designed in such a way that a decrease in $C D$ intensity takes place whenever the concentration of free antigen increases, resulting in a decrease in the concentration of aggregates. In comparing CD absorption curves of the probe in the presence of non-allergic proteins, the latter does not affect the strong CD intensity of the probe, indicating the specificity of the biosensor[83].

The deposition of gold or shells around gold heterodimers gives tuneable $\mathrm{CD}$ signals, which depends on the composition, shell thickness and sequence of metallic layer (Fig. 14). The chiroptical assemblies of nanoparticles produce amplified chiroptical signal, were used for DNA detection at zeptomolar level. The chiroplasmonic band of $\mathrm{Au}$ heterodimers (HDs) at $525 \mathrm{~nm}$ exhibited a red shift pink to purple by an Au shell (@Au) deposition on it. The strong peak at $581 \mathrm{~nm}$ of HD@Au gets amplified with a g-factor of $1.21 \times 10^{-2}$. The formation of shell on HDs decreased the gap between two nanoparticles thereby enhanced the $\mathrm{CD}$ bands. The narrow CD spectra with the highest $\mathrm{g}$-factor is obtained in HD@Au with g-factor 1.03 $\times 10^{-2}$ and doubleshelled Ag shell followed by Au shell on HD (HD@Ag@Au) with $\mathrm{g}$-factor $0.44 \times 10^{-2}$ or Au shell followed by Ag shell on HD (HD@Au@Ag) with g-factor $0.28 \times 10^{-2}$. The strong CD bands of HD@Au were utilized for DNA detection. The DNA concentration quantitatively affects the HD@Au and optical activity of the resulting dispersions. The LOD of the HD@Au-based biosensor was low (Table 1) as compared to fluorometric QD assay ( $\mathrm{LOD}=1 \mathrm{aM})$ [84] and colorimetric biobar-code assay (LOD $=500 \mathrm{zM}$ ) [85]. Here, the enhanced $\mathrm{CD}$ signal in combination with polymeric chain reaction is attributed to the highly sensitive detection of DNA [86].

The Ag NP-Au NP heterodimers linked with antigen-antibody bridges exhibit chiroplasmonic properties. These dimers with scissor-like conformation were utilized for the bioanalysis of a cancer biomarker prostate-specific antigen and environmental pollutant microcystin-LR (Fig. 15). Here, the Au NPs and Ag NPs were modified with anti-microcystin-LR (MCLR) corresponding antibodies and BSA-antimicrocystin-LR, respectively, mixed together so that bridged heterodimers were formed. These heterodimers produce two prominent CD signals. The existence of plasmonic nanoparticle enhanced the chirality via coupling of angled conformation with circularly polarized light. The advantage of this study over previously studied cases is the explanation of the origin of chiral signals in heterodimers. The occurrence of chirality in bridged dimers depends on dihedral angle, $\theta$. The toxin MCLR results in the formation of dimers, which exhibit bisignate CD signal between $526 \mathrm{~nm}$ ad $399 \mathrm{~nm}$. The LOD (Table 1) for MCLR is 250 times lower as compared to ELISA assay. In addition to this, the chiroplasmonic method was extended to the detection of prostate-specific antigen. The intensity of CD signal increases with concentration of PSA because of the assembly of heterodimers [87].

Plasmonic Au NPs were self-assembled with lanthanide-doped upconversion nanoparticles in the proximity of suitable DNA frame. The assembled pyramids exhibit 
Fig. 14 Schematic illustration of a PCR-assembled HDs and b sition of Ag and Au shell(s). "Reprinted with permission from Ref. 86. Copyright (2014) American Chemical Society" sequential post-assembly depo-

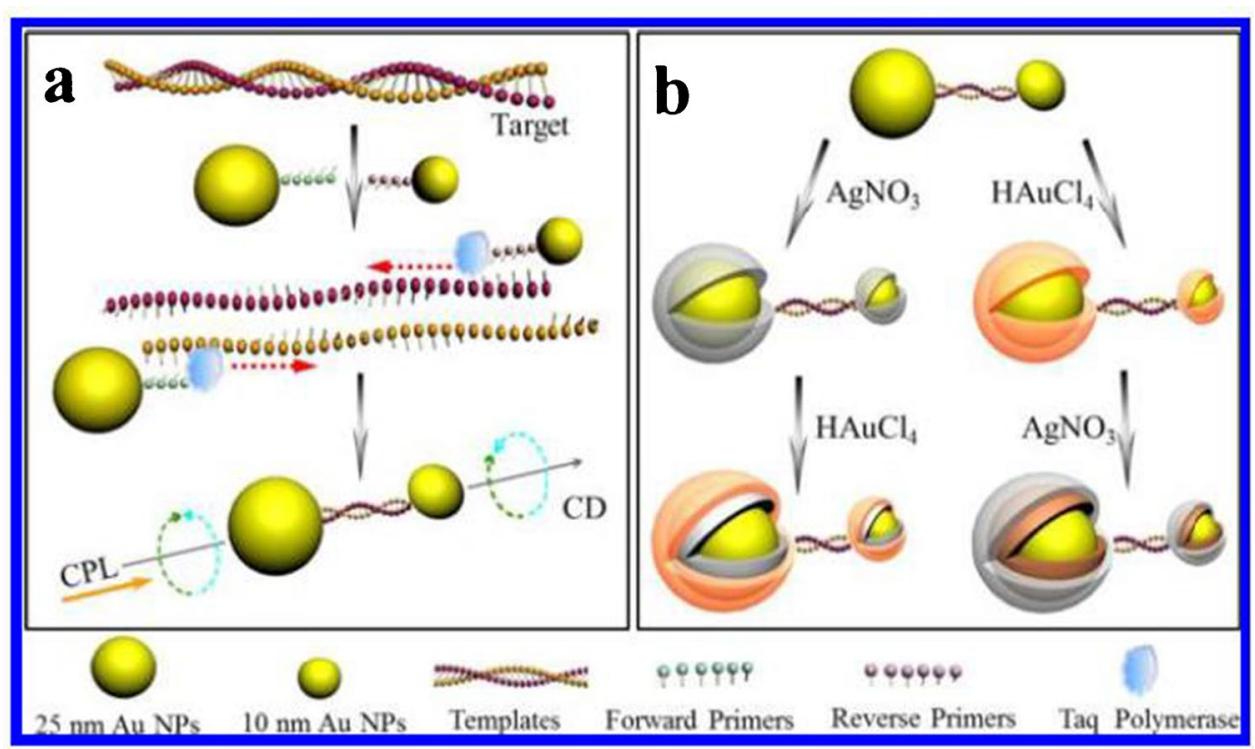

Fig. 15 Schematic illustration for the assembly of NP hetero dimers and their use for biological analysis. A, B The NP dimer was assembled from $\mathrm{Au}$ NPs and Ag NPs, which were functionalized with complementary biomacromolecules (A). For the detection of small peptides, exemplified by MCLR, the competitive immunorecognition assay was chosen to demonstrate its applicability to biological analysis. It results in a decrease of the CD amplitude (B). For detection of the fairly large proteins, exemplified by PSA, we used sandwich immunoassay mode. C Schematics of the NP dimers bridged by immunocomplexes used in competitive and sandwich immunoassays. "Reprinted with permission from Ref. 87. Copyright (2013) American Chemical Society"
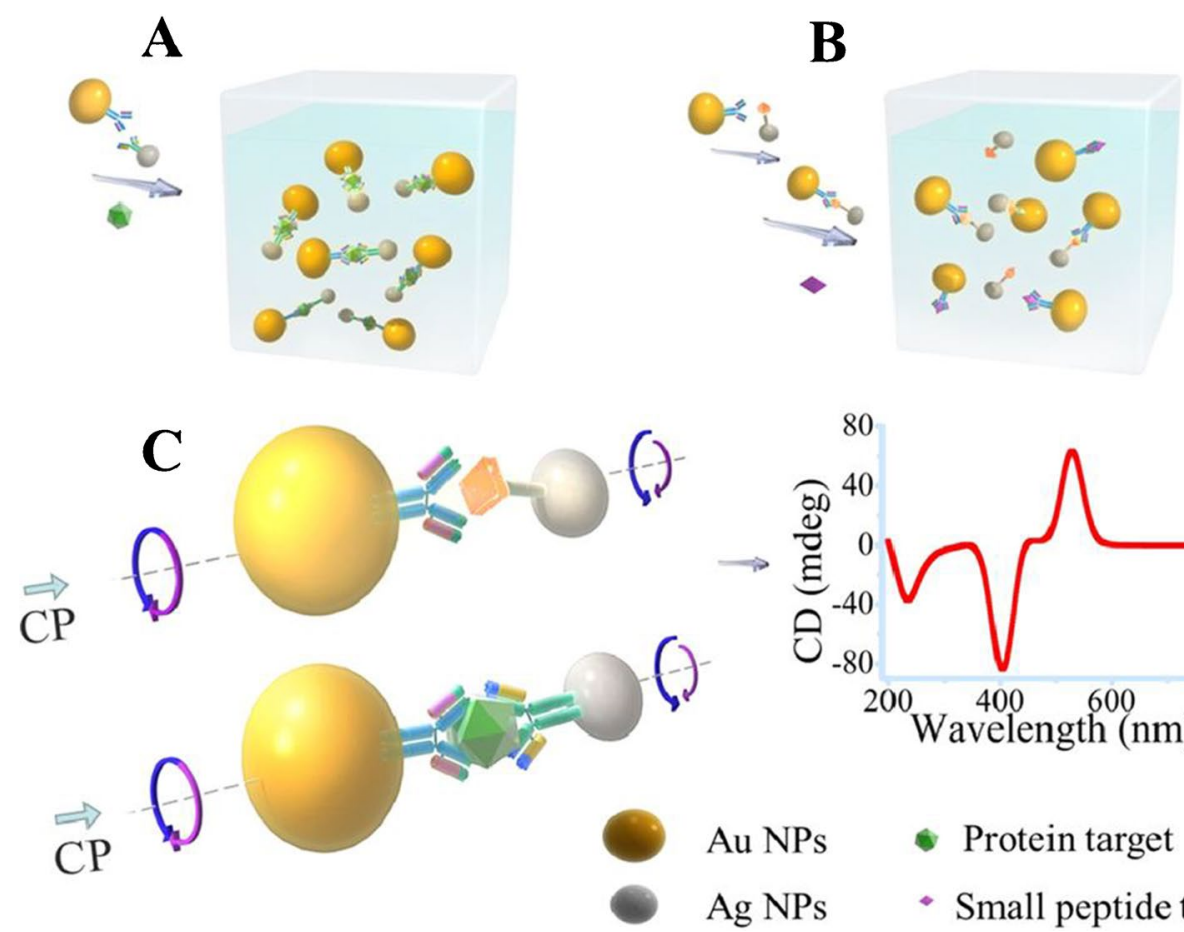

\section{Au NPs Protein target \\ Ag NPs - Small peptide target}

chiroptical properties in the visible region, whereas the luminescence of upconversion nanoparticles get quenched by the LRET [88]. In the presence of miRNA, the DNA frame undergoes complete dissociation resulting in the separation of Au NPs and upconversion nanoparticle. The chiroplasmonic DNA-driven gold-upconversion nanopyramids exhibit dual signals of strong $\mathrm{CD}$ at $521 \mathrm{~nm}$ and remarkable luminescence in 500-600 $\mathrm{nm}$. The nanoprobe was applied for the real-time detection of intracellular micro-RNA in living cells (Table 1 ). The intensity of CD spectra gets decreased, whereas that of fluorescence gets increased as the concentration of miR-21 gets increased in the cells. The sensitivity of $\mathrm{CD}$ detection is 4 times stronger than that of fluorescence detection. The $\mathrm{CD}$ signals are more prominent than luminescent signals, owing to the plasmonic enhancement via intrinsic chirality of DNA molecule and spin angular momentum of photon of the nanopyraminds [32].

A chiral-aptasensor based on Au NP heterodimer could be used to quantify intracellular ATP levels. The chiral nanoassembly exhibit special CD signal with high background intensity and avoid optical interference with autofluorescence of cellular organelles. The decay of CD peak 
of heterodimers at $523 \mathrm{~nm}$ gets enhanced in the presence of ATP, owing to the chiroplasmonic detection of ATP. This type of chiral Au NP sensors can be explored to monitor and investigate biological activity in living organisms [89].

The chiral Au@AuAg yolk-shell nanorods were synthesized using chiral D- and L-penicillamines. The circular dichroism of plasmonic core-satellite superstructures depend on concentration of chiral molecules, hot spots in the nanogaps of Au NRs, aspect ratio of Au NRs and coulombic interaction between Au NRs and D- or L-penicillamines. This superstructure with increased $\mathrm{g}$-factor (0.021) and strong plasmonic CD intensity was employed as the photothermal probes for the quantitative detection of zinc ions in Parkinson's cells (human SH-SY5Y cells). Here, the CD response of the nanoassembly originates from the coulombic interaction between Au NR and D/Lpenicillamines. The plasmonic $\mathrm{CD}$ also gets enhanced due to the formation of hot spots created between nanogaps in the nanoassembly. The biosensor is designed in such a way that the CD response of the nanoasembly at $530 \mathrm{~nm}$ and $740 \mathrm{~nm}$ decreases with increase in concentration of $\mathrm{Zn}^{2+}$ ion in cells, suggesting the disassembly of the nanostructure via the dissociation of DNAzyme by $\mathrm{Zn}^{2+}$ ions. The nanoassembly shows good selectivity over other metal ions, negligible cytotoxicity and its $\mathrm{CD}$ response has no interference with proteins or peptides[90].

The chiroplasmonic active optical fibre probe was assembled with gold and silver layers followed by immobilization of helicene enantiomers. The nanoassembly was able to produce chiroplasmonic waves via chirality transfer for helicene to plasmonic bands. The helicene used were rac-9-(3-pyridylethylnyl)[7]helicene, rac-9-(4-pyridylethynyl)[7]helicene and racemic 2-(3-thienyl)[6]helicene. The complete information of a molecule in the solution was analysed with "cross" functionalization of the helicene enantiomers. The nanoassembly was applied for the detection of $\beta$-lactoglobulin and glucose enantiomers. The probe works on the sensitive interaction of chiral plasmons with chiral environment [91]. The sensitivity is greater for conformation chirality possessed by $\beta$-lactoglobulin as compared to point chirality in glucose enantiomers. The achiral plasmonic Au NP-based substrate was used to detect the action of scorpion venom in human's blood serum. A change in concentration of the venom casue a variation in the refractive index of the substrate. The CD signal depends on the lattice plasmon polaritons. It creates an action potential at the sample surface, which is unique for a particular neurotoxin [31].

\section{Biodetection}

A chiral assembly of sugammadex-Au NPs was used as a colorimetric chiral sensor for detecting lysine and asparagine enantiomers. In addition to this, it exhibits excellent catalytic activity towards the reduction of 4-nitrophenol with sodium borohydride [92]. The two-dimensional chiral arrangements of gold nanoprisms give strong second harmonic generation chiroptical effects, where chirality originates through inter-particle plasmonic interactions and mutual arrangement. These "meta-molecules" could aid as building blocks for a novel "watermark", which could be read-out encoded pattern by means of a sophisticated nonlinear microscopy technique. The chiral N-acetyl-L-cysteine-modified Au NPs were employed as a chiral selector for the enantioselective recognition of S-naproxen and R-naproxen[93].

The 3.3-nm chiral L-glutathione and D-glutathionecapped Au NPs named as L3.3 and D3.3, respectively, were used for the chiral recognition of amyloid beta peptide and inhibition of $A \beta 42$ fibrillation occurs in Alzheimer's diseases. The inhibition activity of A $\beta 42$ fibrillization was greatly enhanced by glutathione stabilized Au NPs as compared to pristine glutathione molecules. The conformational changes associated with the interaction of AuNPs with A $\beta 42$ were analysed using $\mathrm{CD}$ spectroscopy. The positive $\mathrm{CD}$ band at $195 \mathrm{~nm}$ and negative CD peak at $215 \mathrm{~nm}$ in the presence of D3.3 give a distinguished effect as compared to L3.3. It manifests the dependence of surface chirality of glutathionecoated Au NPs. Here, the Au NP prevents the structural change of $A \beta 42$ from its bare conformation to $\beta$-sheet conformation in solution. The chiral glutathione-stabilized $\mathrm{Au}$ NPs have opened a new era for bioanalysis toward the fatal Alzheimer's diseases [94]. The L- and D-glutathione can act as a reducing agent for the synthesis of gold nanoclusters (Au NCs.) The positive surface charge of Au NCs is attained by using the cationic ligand, 4,6-diamino-2-pyrimidinethiol. The CD spectra of both L- and D-glutathione-based Au NCs consist of mirrored CD peaks. The D-glutathione-protected $\mathrm{Au}$ NCs are less toxic to cells as well as organs. Thus, the application of it in in vivo is safer than its L-glutathionebased counterparts. The study was extended into mice and identified the superiority of D-form over L-form [95]. Several stages of studies and modifications are necessary for the application of such nanoassembly in daily life.

The Au NPs were grafted with 2-mercaptoacetyl-L(D)valine (L(D)MAV) and poly(acryloyl-L(D)-valine (L(D)PAV) chiral molecules. The PAV-Au NPs give strong CD signals as compared to MAV-Au NPs because of the presence of a greater number of carboxyl groups in PAV-Au NPs. The mirror image CD spectra of L-PAV-Au NPs and D-PAV-Au NPs appear between 190 and $300 \mathrm{~nm}$ region. The internalization of chiral D-PAV-Au NPs in A549 and HepG2 cells were significantly greater than MAV-Au NPs and L-PAV-Au NPs. This chiral selective interaction arises as a result of preferable interaction between D-PAV molecules on Au NPs and L-phospholipid-based cell membrane [96]. 
The plasmonic hot spots chiroptical activity of DNAbridged chiral Au NPs/Au NRs dimers concede one to monitor the internalization of it in mammalian cells. The $\mathrm{CD}$ peaks in the visible region get reversed from negative to positive owing to the variation in the conditions of the dimers (Fig. 16a) inside and outside the cervical cancer HeLa cells, which is in good agreement with simulated CD spectra. The chirality reversal arises due to the variation in the elastic, electrostatic and van der Waals interactions resulting in the conformational changes of DNA-bridged $\mathrm{Au}$ NR dimers. The image from biological transmission electron microscopy (bio-TEM) proved that the dimers are dispersed in the cytosol of HeLa cells (Fig. 16b). Cryo-TEM tomography and its statistical analysis showed that the chirality of the dimers transformed from right-handed to left-handed conformation: the average dihedral angles are $-12.5 \pm 2^{\circ}$ (inside) and $+9.6 \pm 1^{\circ}$ (outside) of the cells (Fig. 16c and d). The chiroplasmonic gold nanostructures can be used to extend its contribution in photodynamic therapy and quantitative estimation of nanoscale interactions [97].

The chiral Au NPs with high polarizability can interact with circularly polarized light (CPL), which may induce stringent cellular response to chiral photons. The Au NPs stabilized with $\mathrm{D} / \mathrm{L}$-cysteine and functionalized with single-stranded DNA (ssDNA). The DNA-bridged chiral Au NPs were established for the distinction of neural stem cells (NCS) into neurons with the aid of CPL. The nanoassemblies enter the stem cells and trigger conformational changes, entangled with actin nanofibres. The nanoparticle denoted as $\mathrm{C}_{30}(\mathrm{D}) \mathrm{S}_{5}-\mathrm{C}_{20}(\mathrm{~L})$ or $\mathrm{C}_{30}(\mathrm{~L}) \mathrm{S}_{5}-\mathrm{C}_{20}(\mathrm{D})$ is fabricated by connecting 5 -nm dangling nanoparticle with DNA-functionalized D- or L-cysteine-capped Au NPs having $30 \mathrm{~nm}$ or $20 \mathrm{~nm}$ (L- and D- stands for handedness of cysteine). The $\mathrm{C}_{30}(\mathrm{D}) \mathrm{S}_{5}-\mathrm{C}_{20}(\mathrm{~L})$ nanoassemblies with a negative $C D$ peak at $524 \mathrm{~nm}$ reconfigure to as $\mathrm{C}_{30}(\mathrm{D})-\mathrm{C}_{20}(\mathrm{~L}) \mathrm{S}_{5}$ in the presence of Fox $3 m$ RNA with a positive $\mathrm{CD}$. The CD of the nanoassemblies originates from the mirror asymmetry
Fig. 16 Chiral geometry of NP dimers. a TEM image of NP dimers in cell culture media; scale bar, $100 \mathrm{~nm}$. b Bio-TEM images of NP dimers in the HeLa cells; scale bars, $100 \mathrm{~nm}$. c TEM tomography images (bottom) of NP dimers both outside and inside cells with schematics of dimers' geometry (top). d Statistical analysis of the dihedral angles $\theta$ for NP dimers inside and outside the cell as determined from cryo-TEM tomography images. The error bars correspond to the standard error of the mean $(n=3)$. The sign of the dihedral angle in these nanoscale structures was chosen in accord with the IUPAC convention. e Simulated CD spectra of NP dimers intra- and extracellular localization of NP dimers based on geometries from d. " Reproduced with permission from Ref. 97. Copyright (C) 2017, Springer Nature"
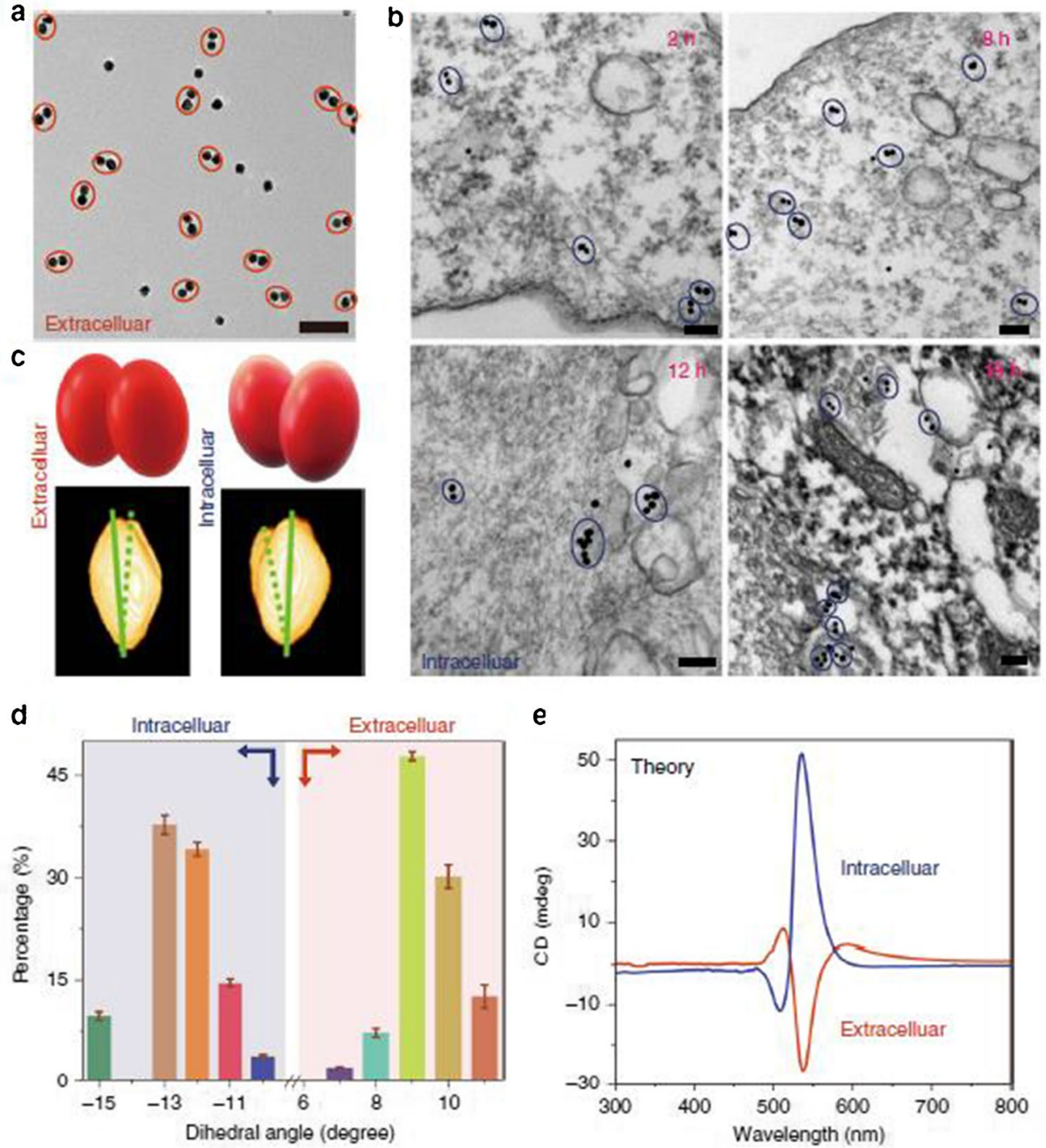

e

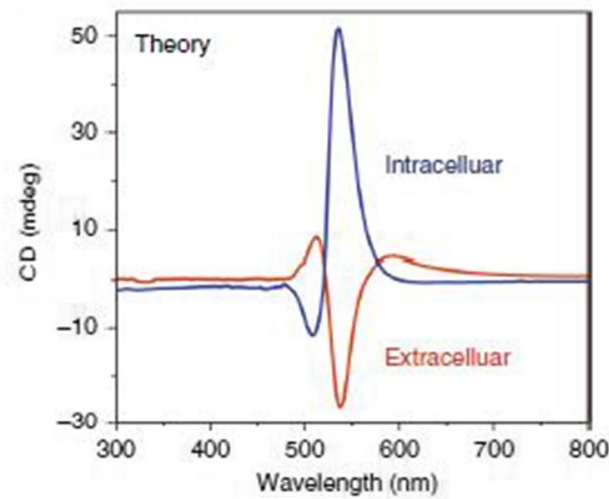


of the nanoassembly [98][98]. The CPL impose polarization-reliant constrain on the nanofibres via nanoassemblies, owing to the differentiation of NCS. The biological effects of CPL based on Au NP-based nanoassemblies can be used for further exploration in bio detection applications [99].

The gold Janus nanoparticles capped with hydrophobic hexanethiolate ligands on one face and hydrophilic 3-mercapto-1,2-propanediol on the other face of the core. This Janus nanoparticle self-assembled into hollow vesicle-like nanostructures in both aqueous and organic phases. It exhibits circular dichroism absorption in the visible region, where the intensity of $\mathrm{CD}$ increases with increase in concentration of Au NPs. The gold Janus nanoparticle on interaction with $\mathrm{L}$-alanine gives no change in plasmonic $\mathrm{CD}$, whereas D-alanine shows negative plasmonic CD. The simulation study shows that a vertex formed on the Janus particle, which gives unique nature for it. The chiral vesicle-like $\mathrm{Au}$ NPs possess high enantioselectivity towards D-alanine compared to L-alanine. These types of Janus nanosystem could be extended for the identification, separation and biosensing of various biomolecules [100].

A chiral carbon nanotube electropolymerized with dopamine and loaded with Au NPs on it was utilized for the quantitative and qualitative electrochemical recognition of tryptophan and tyrosine isomers. Here, the dopamine directs the uniform dispersion of Au NPs on carbon nanotubes. The conductivity of Au NPs blended with chirality of carbon nanotube established as a novel chiral electrochemical biosensing system for amino acids [101]. The chiral plasmonic Au NR-based biosensors were employed for the hydrogen bonding $(\mathrm{Hb})$ and zwitterionic electrostatic interactions $(\mathrm{Zw}-$ Es) between amine and carboxylic acid group in aqueous media. Such nanosensors are sensitive to conformations and selective to chirality at the nanoscale interfaces in $\mathrm{Hb}$ and $\mathrm{Zw}$-Es interactions. The SERS technique could be coupled to chiral plasmonic sensors for developing multifunctional biosensors to investigate the chirality in biological systems [102].

\section{Outlook of the bioanalytical applications}

Chiral plasmonic gold nanostructure-based bioassay has been summarized in this section (Table 1). Several enhanced attributes of the chiral plasmonic gold nanostructures, including strong chiroptical effects, tuneable circular dichroism effect, polarization modulation, chiral recognition and handedness dependent plasmonic responses, make them fascinating chiroptical probes for various biosensing and biodetection applications. These platforms may attain accuracy in detection with size, morphology, composition, aspect ratio, nanogap and template-dependent variation in $\mathrm{CD}$ response. Despite the development that occurred in the past decade, chiral plasmonic gold nanostructures are still demanding improvements, which encumbers the biosensing translation of the advanced techniques into daily life. Therefore, it is very important to combine several optical properties of chiral plasmonic nanomaterials including SPR effect, SERS effect and chirality along with CD response. The Au NP-based chiral plasmonic nanomaterial can be synthesized and functionalized with biocompatible surfactants/chiral biomolecules for utilizing it as a bioassay. The synthetic procedures of citrate-stabilized Au NPs are simple, at which DNA template or biomolecules can be tailored to obtain chirality. Also, thiol-based linker molecules can effectively bind on Au NPs so that optimum separation between nanoparticles can be ensured. The rapid development in this field should impart chiral plasmonic Au NP-based probe with enhanced CD effect, well-explained mechanism, better detection limit, biocompatibility, sensitivity and reproducibility.

\section{Conclusion}

Plasmonic nanoparticles utilizing CD spectroscopy have vital role in bioanalytical applications, and therefore, the development of effective biosensors and biodetection probes is of great importance. Bioanalytical tools exhibit importance in various economic background. The progress in this field is forging ahead to offer effective contribution in bioanalytical fields. The requirements for these include the following: (i) High selectivity. For many real samples such as pesticides containing vegetables, enantiomeric drugs, amino acids, peptides, proteins, genetic materials and cells, either broad-spectrum bioanalytical tools capable of unique identification or excellent target-specific probes are necessary. (ii) High sensitivity. The concentration levels of samples are generally below the detection limits of the bioanalytical tools, at which stage the biomolecules are usually undetectable using current platforms. The sensitive bioanalytical sensors allow us to investigate low concentration level of biomolecules. (iii) Low cost. The effectiveness of a bioanalytical probe depends on the extensive use of it among a large population, which is possible only if the probe is cost effective.

Importantly, the development of Au NP-based nanoprobe attains great improvements in bioanalytical field. Among the properties of Au NPs, chiroptical properties have attained much interest due to its high sensitivity and unique features. The interaction of chiral plasmonic Au NPs with electromagnetic radiation generates unique optical signatures. The $\mathrm{CD}$ responses of chiral plasmonic Au NPs depend on the interaction with different biomolecules and are distinctive like a finger print. Different types of chiral gold nanostructure produce a wide range of SPR wavelength. These optically tuneable nanosystems were functionalized with several biomolecules or synthetic molecules in various applications. 
The combination of plasmonic properties and CD response of such nanoassembly could be utilized for fabricating bioanalytical tools.

Although a great progress in Au NP-based chiroptical responsive system has been achieved, many confronting challenges remains. For example, in the case of hot spot formation-based $\mathrm{CD}$ response, the practicability of maintaining distance between plasmonic nanoparticles using linker molecules in a macroscopic system is questionable. Secondly, the detection of large biomolecule-like proteins with the CD spectroscopy using plasmonic nanoparticle is difficult due to the possibility of decay of intensity of electric field between the nanoparticles. In addition to this, the mechanism of CD response is still not clear. Thirdly, the reproducibility of the detection systems is a challenging factor too. Moreover, the application of CD-responsive-based Au NPs in a point of care stage still demands interdisciplinary approach-based research by experts from various fields.

\section{Declarations}

Conflicts of interest The authors declare no competing interests.

\section{References}

1. Banzer P, Woniak P, Mick U, et al (2016) Chiral optical response of planar and symmetric nanotrimers enabled by heteromaterial selection. Nat Commun7:13117.https://doi.org/10.1038/ncomm s13117

2. Wu W, Hu W, Qian G, et al (2019) Mechanical design and multifunctional applications of chiral mechanical metamaterials: a review. Mater Des 180:107950.https://doi.org/10.1016/j.matdes. 2019.107950

3. Cheng X, Miao T, Qian Y, et al (2020) Supramolecular chirality in azobenzene-containing polymer system: traditional postpolymerization self-assembly versus in situ supramolecular selfassembly strategy. International J Mol Sci 21:6186.https://doi. org/10.3390/ijms21176186

4. Inaki M, Liu J, Matsuno K (2016) Cell chirality: its origin and roles in left-right asymmetric development. Philosophical Trans R Soc B: Biol Sci 371:1710.https://doi.org/10.1098/rstb.2015. 0403

5. Chen Y, Ma W (2020) The origin of biological homochirality along with the origin of life. PLOS Comput Biol 16:e1007592.https://doi.org/10.1371/journal.pcbi.1007592

6. Nemati A, Shadpour S, Querciagrossa L, et al (2018) Chirality amplification by desymmetrization of chiral ligand-capped nanoparticles to nanorods quantified in soft condensed matter. Nat Commun 9:3908.https://doi.org/10.1038/s41467-018-06400-0

7. Niinomi H, Sugiyama T, Cheng A-C, et al (2021) Chiral optical force generated by a superchiral near-field of a plasmonic triangle trimer as origin of giant bias in chiral nucleation: a simulation study. J Phys Chem C 125:6209-6221.https://doi.org/10.1021/ acs.jpcc.0c11109

8. Rafiei Miandashti A, Khosravi Khorashad L, Kordesch ME, et al (2020) Experimental and theoretical observation of photothermal chirality in gold nanoparticle helicoids. ACS Nano 144188-4195.https://doi.org/10.1021/acsnano.9b09062

9. Wu T, Wang R, Zhang X (2015) Plasmon-induced strong interaction between chiral molecules and orbital angular momentum of light. Sci Rep 5:18003.https://doi.org/10.1038/srep18003

10. Snyder JK (1996) Circular dichroism: principles and applications K. Nakanishi and N. Berova (Columbia University) and R. W. Woody (Colorado State University). Journal of Natural Products 59:1219. https://doi.org/10.1021/np9604700

11. Chakraborty A, Nonappa, Mondal B, et al (2021) Near-infrared chiral plasmonic microwires through precision assembly of gold nanorods on soft biotemplates. J Phys Chem C 125:32563267.https://doi.org/10.1021/acs.jpcc.0c11512

12. Wang Y, He W, Li CH, et al (2021) Chirality transfer of cysteine to the plasmonic resonance region through silver coating of gold nanobipyramids. Chem Commun 57:32113214.https://doi.org/10.1039/D0CC07576K

13. Ostovar Pour S, Rocks L, Faulds K, et al (2015) Through-space transfer of chiral information mediated by a plasmonic nanomaterial. Nat Chem 7:591-596.https://doi.org/10.1038/nchem. 2280

14. Zhu M, Qian H, Meng X, et al (2011) Chiral $\mathrm{Au}_{25}$ Nanospheres and nanorods: synthesis and insight into the origin of chirality. Nano Lett 11:3963-9.https://doi.org/10.1021/n1202288j

15. Auguié B, Alonso-Gómez JL, Guerrero-Martínez A, LizMarzán LM (2011) Fingers crossed: optical activity of a chiral dimer of plasmonic nanorods. J Phys Chem Lett 2:846851.https://doi.org/10.1021/jz200279x

16. Vinegrad E, Vestler D, Ben-Moshe A, et al (2018) Circular dichroism of single particles. ACS Photonics 5:21512159.https://doi.org/10.1021/acsphotonics.8b00016

17. Smith KW, Zhao H, Zhang H, et al (2016) Chiral and achiral nanodumbbell dimers: the effect of geometry on plasmonic properties. ACS Nano 10:6180-6188.https://doi.org/10.1021/ acsnano.6b02194

18. Sen S, Dasgupta S, DasGupta S (2017) Does surface chirality of gold nanoparticles affect fibrillation of HSA? J Phys Chem C 121:18935-18946.https://doi.org/10.1021/acs.jpcc.7b05354

19. Mullins S-M, Weissker H-Ch, Sinha-Roy R, et al (2018) Chiral symmetry breaking yields the I-Au60 perfect golden shell of singular rigidity. Nat Commun 9:3352.https://doi.org/10.1038/ s41467-018-05215-3

20. Lu J, Chang Y-X, Zhang N-N, et al (2017) Chiral plasmonic nanochains via the self-assembly of gold nanorods and helical glutathione oligomers facilitated by cetyltrimethylammonium bromide micelles. ACS Nano 11:3463-3475.https://doi.org/10. 1021/acsnano.6b07697

21. Merg AD, Boatz JC, Mandal A, et al (2016) Peptide-directed assembly of single-helical gold nanoparticle superstructures exhibiting intense chiroptical activity. J Am Chem Soc 138:13655-13663.https://doi.org/10.1021/jacs.6b07322

22. Zhou X, Liu Q, Shi X, et al (2021) Effect of aspect ratio on the chirality of gold nanorods prepared through conventional seedmediated growth method. Analytica Chimica Acta 1152.https:// doi.org/10.1016/j.aca.2021.338277

23. Wang Z-Y, Zhang N-N, Li J-C, et al (2021) Serum albumin guided plasmonic nanoassemblies with opposite chiralities. Soft Matter 17:6298-6304.https://doi.org/10.1039/D1SM00784J

24. Cho NH, Byun GH, Lim Y-C, et al (2020) Uniform chiral gap synthesis for high dissymmetry factor in single plasmonic gold nanoparticle. ACS Nano 143595-3602.https://doi.org/10.1021/ acsnano.9b10094

25. Slocik JM, Govorov AO, Naik RR (2011) Plasmonic circular dichroism of peptide-functionalized gold nanoparticles. Nano Lett 11701-705.https://doi.org/10.1021/nl1038242 
26. Xu L, Hao C, Yin H, et al (2013) Plasmonic Core-satellites nanostructures with high chirality and bioproperty. J Phys Chem Lett 4:2379-2384.https://doi.org/10.1021/jz401014b

27. Sharma A, Mori T, Lee H-C, et al (2014) Detecting, visualizing, and measuring gold nanoparticle chirality using helical pitch measurements in nematic liquid crystal phases. ACS Nano 8:11966-11976.https://doi.org/10.1021/nn504980w

28. Sun X, Wang N, He Y, et al (2021) Molecule-specific vibrationbased chiral differentiation of Raman spectra using cysteine modified gold nanoparticles: the cases of tyrosine and phenylalanine. J Mater Chem B9:7167-7171.https://doi.org/10.1039/ D1TB00983D

29. Bhat SA, Rao DSS, Prasad SK, Yelamaggad C v. (2021) Chiral plasmonic liquid crystal gold nanoparticles: self-assembly into a circular dichroism responsive helical lamellar superstructure. Nanoscale Advances 3:2269-2279.https://doi.org/10.1039/ D0NA01070G

30. Guerrero-Martínez A, Auguié B, Alonso-Gómez JL, et al (2011) Intense optical activity from three-dimensional chiral ordering of plasmonic nanoantennas. Angewandte Chemie International Edition 50:5499-5503.https://doi.org/10.1002/anie.201007536

31. Wang M, Huang Z, Salut R, et al (2021) Plasmonic helical nanoantenna as a converter between longitudinal fields and circularly polarized waves. Nano Lett 21:3410-3417.https://doi.org/10. 1021/acs.nanolett.0c04948

32. Li S, Xu L, Ma W, et al (2016) Dual-mode ultrasensitive quantification of MicroRNA in living cells by chiroplasmonic nanopyramids self-assembled from gold and upconversion nanoparticles. J Am Chem Soc 138:306-312.https://doi.org/10.1021/jacs.5b103 09

33. Lan X, Zhou X, McCarthy LA, et al (2019) DNA-enabled chiral gold nanoparticle-chromophore hybrid structure with resonant plasmon-exciton coupling gives unusual and strong circular dichroism. J Am Chem Soc 141:19336-19341.https://doi.org/ 10.1021/jacs.9b08797

34. Cai J, Hao C, Sun M, et al (2018) Chiral shell core-satellite nanostructures for ultrasensitive detection of mycotoxin. Small 14:1703931.https://doi.org/10.1002/smll.201703931

35. Nguyen L, Dass M, Ober MF, et al (2020) Chiral assembly of gold-silver core-shell plasmonic nanorods on dna origami with strong optical activity. ACS Nano 14:7454-7461.https://doi.org/ 10.1021/acsnano.0c03127

36. Wang X, Wang Y, Zhu J, Xu Y (2014) Hierarchical AgNR@ Cys@AuNPs helical core-satellite nanostructure: shape-dependent assembly and chiroptical response. J Phys Chem C 118:57825788.https://doi.org/10.1021/jp410620b

37. Meng D, Li X, Gao X, et al (2021) Constructing chiral gold nanorod oligomers using a spatially separated sergeants-andsoldiers effect. Nanoscale 13:9678-9685.https://doi.org/10.1039/ D1NR01458G

38. Shen X, Song C, Wang J, et al (2012) Rolling up gold nanoparticle-dressed dna origami into three-dimensional plasmonic chiral nanostructures. J Am Chem Soc 134:146-149.https://doi.org/10. 1021/ja209861x

39. Martens K, Binkowski F, Nguyen L, et al (2021) Long- and short-ranged chiral interactions in DNA-assembled plasmonic chains. Nat Commun 12:2025.https://doi.org/10.1038/ s41467-021-22289-8

40. Dai G, Lu X, Chen Z, et al (2014) DNA origami-directed, discrete three-dimensional plasmonic tetrahedron nanoarchitectures with tailored optical chirality. ACS Applied Materials \& Interfaces 6:5388-92.https://doi.org/10.1021/am501599f

41. Shen X, Asenjo-Garcia A, Liu Q, et al (2013) Three-dimensional plasmonic chiral tetramers assembled by dna origami. Nano Lett 13:2128-33.https://doi.org/10.1021/nl400538y
42. Zhu C, Wang M, Dong J, et al (2018) Modular assembly of plasmonic nanoparticles assisted by DNA origami. Langmuir 34:14963-14968.https://doi.org/10.1021/acs.langmuir.8b01933

43. Cecconello A, Kahn JS, Lu C-H, et al (2016) dna scaffolds for the dictated assembly of left-/right-handed plasmonic Au NP helices with programmed chiro-optical properties. Journal of the American Chemical Society 138:9895-9901.https://doi.org/ 10.1021/jacs.6b04096

44. Schreiber R, Luong N, Fan Z, et al (2013) Chiral plasmonic DNA nanostructures with switchable circular dichroism. Nat Commun 4:2948.https://doi.org/10.1038/ncomms3948

45. Zhu J, Wu F, Han Z, et al (2021) Strong light-matter interactions in chiral plasmonic-excitonic systems assembled on DNA origami. Nano Lett 21:3573-3580.https://doi.org/10.1021/acs.nanol ett.1c00596

46. Cheng G, Xu D, Lu Z, Liu K (2019) Chiral self-assembly of nanoparticles induced by polymers synthesized via reversible addition-fragmentation chain transfer polymerization. ACS Nano13:1479-1489.https://doi.org/10.1021/acsnano.8b07151

47. Lu X, Song D, Ribbe A, Watkins JJ (2017) Chiral arrangements of $\mathrm{Au}$ nanoparticles with prescribed handedness templated by helical pores in block copolymer films. Macromolecules 50:5293-5300.https://doi.org/10.1021/acs.macromol.7b01364

48. Zhang Q, Hernandez T, Smith KW, et al (2019) Unraveling the origin of chirality from plasmonic nanoparticle-protein complexes. Science 365:1475-1478.https://doi.org/10.1126/science. aax 5415

49. Kim J-Y, Yeom J, Zhao G, et al (2019) Assembly of gold nanoparticles into chiral superstructures driven by circularly polarized light. J Am Chem Soc 141:11739-11744.https://doi.org/10.1021/ jacs.9b00700

50. Lee H-E, Ahn H-Y, Mun J, et al (2018) Amino-acid- and peptidedirected synthesis of chiral plasmonic gold nanoparticles. Nature 556:360-365.https://doi.org/10.1038/s41586-018-0034-1

51. Lee H-E, Kim RM, Ahn H-Y, et al (2020) Cysteine-encoded chirality evolution in plasmonic rhombic dodecahedral gold nanoparticles. Nat Commun 11:263.https://doi.org/10.1038/ s41467-019-14117-x

52. Krishnadas KR, Sementa L, Medves M, et al (2020) Chiral functionalization of an atomically precise noble metal cluster: insights into the origin of chirality and photoluminescence. ACS Nano 14:9687-9700.https://doi.org/10.1021/acsnano.0c01183

53. Dianat A, Gutierrez R, Alpern H, et al (2020) Role of exchange interactions in the magnetic response and intermolecular recognition of chiral molecules. Nano Lett 20:7077-7086.https://doi. org/10.1021/acs.nanolett.0c02216

54. Plum E, Liu X-X, Fedotov VA, et al (2009) Metamaterials: optical activity without chirality. Phys Rev Lett 102:113902.https:// doi.org/10.1103/PhysRevLett.102.113902

55. Hu L, Huang Y, Pan L, Fang Y (2017) Analyzing intrinsic plasmonic chirality by tracking the interplay of electric and magnetic dipole modes. Sci Rep 7:11151.https://doi.org/10.1038/ s41598-017-11571-9

56. Plum E (2016) Extrinsic chirality: Tunable optically active reflectors and perfect absorbers. Appl Phys Lett 108:241905.https:// doi.org/10.1063/1.4954033

57. Yin S, Ji W, Xiao D, et al (2019) Intrinsically or extrinsically reconfigurable chirality in plasmonic chiral metasurfaces. Optics Communications 448:10-14.https://doi.org/10.1016/j.optcom. 2019.05.006

58. Plum E, Fedotov VA, Zheludev NI (2011) Asymmetric transmission: a generic property of two-dimensional periodic patterns. J Opt 13:024006.https://doi.org/10.1088/2040-8978/13/2/024006

59. Hakkarainen T, Petronijevic E, Rizzo Piton M, Sibilia C (2019) Demonstration of extrinsic chirality of photoluminescence with 
semiconductor-metal hybrid nanowires. Sci Rep 9:5040.https:// doi.org/10.1038/s41598-019-41615-1

60. Lu X, Wu J, Zhu Q, et al (2014) Circular dichroism from single plasmonic nanostructures with extrinsic chirality. Nanoscale 6:14244-14253.https://doi.org/10.1039/C4NR04433A

61. Bochenkov VE, Klös G, Sutherland DS (2017) Extrinsic chirality of non-concentric plasmonic nanorings. Optical Materials Express 7:3715-3721.https://doi.org/10.1364/OME.7.003715

62. Hu L, Tian X, Huang Y, et al (2016) Quantitatively analyzing the mechanism of giant circular dichroism in extrinsic plasmonic chiral nanostructures by tracking the interplay of electric and magnetic dipoles. Nanoscale 8:3720-3728.https://doi.org/10. 1039/C5NR08527F

63. Hu L, Huang Y, Fang L, et al (2015) Fano resonance assisting plasmonic circular dichroism from nanorice heterodimers for extrinsic chirality. Sci Rep 5:16069.https://doi.org/10.1038/ srep16069

64. Tian X, Fang Y, Sun M (2015) Formation of enhanced uniform chiral fields in symmetric dimer nanostructures. Scientific Reports 5:17534.https://doi.org/10.1038/srep17534

65. Song C, Blaber MG, Zhao G, et al (2013) Tailorable plasmonic circular dichroism properties of helical nanoparticle superstructures. Nano Lett 133256-61.https://doi.org/10.1021/n14013776

66. Govorov AO, Fan Z, Hernandez P, et al (2010) Theory of circular dichroism of nanomaterials comprising chiral molecules and nanocrystals: plasmon enhancement, dipole interactions, and dielectric effects. Nano Lett 10:1374-1382.https://doi.org/ $10.1021 / \mathrm{nl} 100010 \mathrm{v}$

67. Liu W, Zhu Z, Deng K, et al (2013) Gold nanorod@chiral mesoporous silica core-shell nanoparticles with unique optical properties. J Am Chem Soc 135:9659-9664.https://doi.org/10. $1021 / \mathrm{ja} 312327 \mathrm{~m}$

68. Yin X, Schäferling M, Metzger B, Giessen H (2013) Interpreting chiral nanophotonic spectra: the plasmonic Born-Kuhn model. Nano Lett 13:6238-6243.https://doi.org/10.1021/nl403705k

69. Gui L, Hentschel M, Defrance J, et al (2019) Nonlinear BornKuhn analog for chiral plasmonics. ACS Photonics 6:33063314.https://doi.org/10.1021/acsphotonics.9b01400

70. Mokashi-Punekar S, Walsh TR, Rosi NL (2019) Tuning the structure and chiroptical properties of gold nanoparticle single helices via peptide sequence variation. J Am Chem Soc 141:15710 15716.https://doi.org/10.1021/jacs.9b08798

71. Nemati A, Shadpour S, Querciagrossa L, et al (2019) Highly sensitive, tunable chirality amplification through space visualized for gold nanorods capped with axially chiral binaphthyl derivatives. ACS Nano 13:10312-10326.https://doi.org/10.1021/acsna no. $9 \mathrm{~b} 03787$

72. Ma Z, Gao G, Luo Z, et al (2019) Tuning chirality transfer and amplification of supraparticles via solvent inducing self-aggregation of chiral gold nanoclusters. J Phys Chem C 123:2497324978.https://doi.org/10.1021/acs.jpcc.9b08063

73. Mori T, Sharma A, Hegmann T (2016) Significant enhancement of the chiral correlation length in nematic liquid crystals by gold nanoparticle surfaces featuring axially chiral binaphthyl ligands. ACS Nano 10:1552-1564.https://doi.org/10.1021/acsnano.5b071 64

74. Zhu L, Li X, Wu S, et al (2013) Chirality control for in situ preparation of gold nanoparticle superstructures directed by a coordinatable organogelator. Journal of the American Chemical Society 135: 9174-9180.https://doi.org/10.1021/ja403722t

75. Oh HS, Liu S, Jee H, et al (2010) Chiral poly(fluorene-alt-benzothiadiazole) (pfbt) and nanocomposites with gold nanoparticles: plasmonically and structurally enhanced chirality. J Am Chem Soc 132:17346-17348.https://doi.org/10.1021/ja107064a
76. Yoo S, Park Q-H (2015) Enhancement of chiroptical signals by circular differential Mie scattering of nanoparticles. Sci Rep 5:14463.https://doi.org/10.1038/srep14463

77. Ha J-M, Solovyov A, Katz A (2009) Postsynthetic modification of gold nanoparticles with calix[4]arene enantiomers: origin of chiral surface plasmon resonance. Langmuir 25:153-158.https:// doi.org/10.1021/la802665t

78. Sunaga N, Haraguchi T, Akitsu T (2019) Orientation of chiral schiff base metal complexes involving Azo-groups for induced cd on gold nanoparticles by polarized uv light irradiation. Symmetry 11:1094.https://doi.org/10.3390/sym11091094

79. Bera K, Ghosh T, Basak S (2015) Synthesis of chiral, crystalline Au-nanoflower catalyst assisting conversion of rhodamineB to rhodamine-110 and a single-step, one-pot, eco-friendly reduction of nitroarenes. The Journal of Physical Chemistry C 119:1800-1808.https://doi.org/10.1021/jp5086125

80. Kumagai PS, DeMarco R, Lopes JLS (2017) Advantages of synchrotron radiation circular dichroism spectroscopy to study intrinsically disordered proteins. Eur Biophys J 46:599-606. https://doi.org/10.1007/s00249-017-1202-1

81. Miles AJ, Janes RW, Wallace BA (2021) Tools and methods for circular dichroism spectroscopy of proteins: A tutorial review. Chem Soc Rev 50:8400-8413

82. Ahmed SR, Nagy É, Neethirajan S (2017) Self-assembled star-shaped chiroplasmonic gold nanoparticles for an ultrasensitive chiro-immunosensor for viruses. RSC Adv 7:4084940857.https://doi.org/10.1039/C7RA07175B

83. Wang Y, Rao Z, Zhou J, et al (2019) A chiral assembly of gold nanoparticle trimer-based biosensors for ultrasensitive detection of the major allergen tropomyosin in shellfish. Biosens Bioelectron 132:84-89.https://doi.org/10.1016/j.bios.2019.02. 038

84. Zhou J, Wang Q, Zhang C (2013) Liposome-quantum dot complexes enable multiplexed detection of attomolar DNAs without target amplification. J Am Chem Soc 135:20562059.https://doi.org/10.1021/ja3110329

85. Nam J-M, Stoeva SI, Mirkin CA (2004) Bio-Bar-Codebased DNA detection with PCR-like sensitivityJ. Am. Chem. Soc.126:5932-5933.https://doi.org/10.1021/ja049384

86. Zhao Y, Xu L, Ma W, et al (2014) Shell-engineered chiroplasmonic assemblies of nanoparticles for zeptomolar DNA detection. Nano Lett 14:3908-13.https://doi.org/10.1021/n1501 $166 \mathrm{~m}$

87. Wu X, Xu L, Liu L, et al (2013) Unexpected chirality of nanoparticle dimers and ultrasensitive chiroplasmonic bioanalysis. J Am Chem Soc 135:18629-18636.https://doi.org/10.1021/ja409 5445

88. Li Z, Lv S, Wang Y, et al (2015) Construction of LRET-based nanoprobe using upconversion nanoparticles with confined emitters and bared surface as luminophore. J Am Chem Soc 137:3421-3427.https://doi.org/10.1021/jacs.5b01504

89. Fu P, Sun M, Xu L, et al (2016) A self-assembled chiralaptasensor for ATP activity detection. Nanoscale 8:1500815015.https://doi.org/10.1039/C6NR04086A

90. Hao C, Xu L, Sun M, et al (2018) Chirality on hierarchical selfassembly of Au@AuAg yolk-shell nanorods into core-satellite superstructures for biosensing in human cells. Adv Func Mater 28:1802372.https://doi.org/10.1002/adfm.201802372

91. Miliutina E, Zadny J, Guselnikova O, et al (2021) Chiroplasmon-active optical fiber probe for environment chirality estimation. Sensors and Actuators B: Chemical 343:130122.https:// doi.org/10.1016/j.snb.2021.130122

92. Luo Y, Zhao X, Cai P, Pan Y (2020) One-pot synthesis of an anionic cyclodextrin-stabilized bifunctional gold nanoparticles for visual chiral sensing and catalytic reduction. Carbohydrate 
Polymers 237:237:116127.https://doi.org/10.1016/j.carbpol. 2020.116127

93. Boussouar I, Chen Q, Chen X, et al (2017) single nanochannel platform for detecting chiral drugs. Anal Chem 89: 11101116.https://doi.org/10.1021/acs.analchem.6b02682

94. Hou K, Zhao J, Wang H, et al (2020) Chiral gold nanoparticles enantioselectively rescue memory deficits in a mouse model of Alzheimer's disease. Nat Commun 11:4790.https://doi.org/10. 1038/s41467-020-18525-2

95. Tang H, Li Q, Yan W, Jiang X (2021) Reversing the chirality of surface ligands can improve the biosafety and pharmacokinetics of cationic gold nanoclusters. Angewandte Chemie International Edition 60:3829-13834.https://doi.org/10.1002/anie. 202101609

96. Deng J, Wu S, Yao M, Gao C (2016) Surface-anchored poly(acryloyl-L(D)-valine) with enhanced chirality-selective effect on cellular uptake of gold nanoparticles. Sci Rep 6:31595.https://doi.org/10.1038/srep31595

97. Sun M, Xu L, Bahng JH, et al (2017) Intracellular localization of nanoparticle dimers by chirality reversal. Nat Commun 8:1847.https://doi.org/10.1038/s41467-017-01337-2

98. Milton FP, Govan J, Mukhina MV, Gun'ko YK (2016) The chiral nano-world: chiroptically active quantum nanostructures. Nanoscale Horizons 1:14-26.https://doi.org/10.1039/C5NH0 0072F
99. Qu A, Sun M, Kim J-Y, et al (2021) Stimulation of neural stem cell differentiation by circularly polarized light transduced by chiral nanoassemblies. Nat Biomed Eng 5:103-113.https://doi. org/10.1038/s41551-020-00634-4

100. Lu JE, Yang C-H, Wang H, et al (2018) Plasmonic circular dichroism of vesicle-like nanostructures by the templateless self-assembly of achiral Janus nanoparticles. Nanoscale 10:14586-14593.https://doi.org/10.1039/C8NR05366A

101. Lu H, Wang Z, Fan X, et al (2020) Electrochemical chiral amino acid biosensor based on dopamine-localized gold nanoparticles @ left-handed spiral chiral carbon nanotubes. Analytical Methods 12:3901-3908.https://doi.org/10.1039/D0AY00921K

102. Zhao W, Wang R-Y, Wei H, et al (2017) Recognition of chiral zwitterionic interactions at nanoscale interfaces by chiroplasmonic nanosensors. Phys Chem Chem Phys 19:2140121406.https://doi.org/10.1039/C7CP03004E

Publisher's note Springer Nature remains neutral with regard to jurisdictional claims in published maps and institutional affiliations. 